\title{
LIMIT THEOREMS FOR SOME CONTINUOUS-TIME RANDOM WALKS
}

\author{
M. JARA, ${ }^{*}$ Université de Paris Dauphine and Instituto de Matématica Pura e Aplicada \\ T. KOMOROWSKI,** Uniwersytet Marii Curie-Skłodowskiej and \\ Polish Academy of Sciences
}

\begin{abstract}
In this paper we consider the scaled limit of a continuous-time random walk (CTRW) based on a Markov chain $\left\{X_{n}, n \geq 0\right\}$ and two observables, $\tau(\cdot)$ and $V(\cdot)$, corresponding to the renewal times and jump sizes. Assuming that these observables belong to the domains of attraction of some stable laws, we give sufficient conditions on the chain that guarantee the existence of the scaled limits for CTRWs. An application of the results to a process that arises in quantum transport theory is provided. The results obtained in this paper generalize earlier results contained in Becker-Kern, Meerschaert and Scheffler (2004) and Meerschaert and Scheffler (2008), and the recent results of Henry and Straka (2011) and Jurlewicz, Kern, Meerschaert and Scheffler (2010), where $\left\{X_{n}, n \geq 0\right\}$ is a sequence of independent and identically distributed random variables.
\end{abstract}

Keywords: Continuous-time random walk; Markov chain; subordinator; jump process

2010 Mathematics Subject Classification: Primary 60G50; 60F17

Secondary 60G18

\section{Introduction}

Continuous-time random walks (CTRWs) were introduced in [23] and have applications in the modeling of various phenomena, e.g. in anomalous transport (see, e.g. [9], [13], [26], [27], and [30]), mathematical finance (see [14] and [21]), and in hydrology (see [4] and [5]). A CTRW can be described as a random walk subordinated to a renewal process. More precisely, suppose that $(E, d)$ is a Polish space with $\&$ the $\sigma$-algebra of its Borel subsets, that $\tau: E \rightarrow(0,+\infty)$ and $V: E \rightarrow \mathbb{R}$ are two measurable functions, and that $\left\{X_{n}, n \geq 0\right\}$ is a Markov chain with an initial distribution $\pi$ that is stationary. Suppose also that

$$
t_{0}:=0, \quad t_{N}:=\sum_{k=0}^{N-1} \tau\left(X_{k}\right), \quad N \geq 1,
$$

are the renewal times. Particle jumps are given by $V\left(X_{k}\right), k=0,1, \ldots$ Let $S_{0}:=0$ and $S_{N}:=\sum_{k=0}^{N-1} V\left(X_{k}\right)$ for $N \geq 1$. For any $t \geq 0$, let $n(t):=\max \left[N \geq 0: t_{N} \leq t\right]$. We define a stochastic process describing the trajectory of the particle performing a CTRW by

$$
W(t):=S_{n(t)}, \quad t \geq 0
$$

Received 11 May 2010; revision received 9 March 2011.

* Postal address: CEREMADE, UMR CNRS 7534, Université de Paris Dauphine, Place du Maréchal de Lattre de Tassigny, 75775 Paris Cedex 16, France. Email address: jara@ceremade.dauphine.fr

** Postal address: Institute of Mathematics, Uniwersytet Marii Curie-Skłodowskiej, pl. Marii Curie-Skłodowskiej 1, Lublin 20-031, Poland. Email address: komorow@ hektor.umcs.lublin.pl 
We are concerned with describing the limiting behavior of the scaled processes $\left\{N^{-\gamma} W(N t)\right.$, $t \geq 0\}$ for an appropriate $\gamma>0$ as $N \rightarrow+\infty$.

The issue of describing the limiting behavior of a CTRW based on a Markov chain, as described above, arises naturally in the context of investigating the limit of an additive functional $Y(t):=\int_{0}^{t} \Psi\left(K_{s}\right) \mathrm{d} s$ of a Markovian jump processes $\left\{K_{t}, t \geq 0\right\}$ with phase space $F$. Suppose that the generator of the process is given by

$$
L f(x)=\lambda(x) \int_{F}[f(y)-f(x)] P(x, \mathrm{~d} y), \quad f \in B_{b}(F),
$$

where $B_{b}(F)$ is the space of bounded and Borel measurable functions on $F$, and $P(x, \mathrm{~d} y)$ is a transition probability function of a certain $F$-valued Markov chain $\left\{Z_{n}, n \geq 0\right\}$. The functions $\Psi$ and $\lambda$, defined on $F$, are Borel measurable and $\lambda$ is positive. Consider a Markov chain $\left\{X_{n}:=\right.$ $\left.\left(Z_{n}, \xi_{n}\right), n \geq 0\right\}$, taking values in $E:=F \times(0,+\infty)$, where $\left\{\xi_{n}, n \geq 0\right\}$ is an independent and identically distributed (i.i.d.) sequence of random variables independent of $\left\{Z_{n}, n \geq 0\right\}$ and exponentially distributed with intensity 1 . Then $K_{t}=Z_{n}$ for $t \in\left[t_{n}, t_{n+1}\right)$, where $t_{n}$ is given by (1.1), with $\tau(x, \xi):=\xi \lambda^{-1}(x)$. Consequently, $Y(t)-W(t)=\left(t-t_{n}\right) \Psi\left(Z_{n}\right)$ for $t \in\left[t_{n}, t_{n+1}\right)$, where $W(t)$ is described by (1.2) with $V(x, \xi):=\Psi(x) \tau(x, \xi)$. A somewhat related model of the CTRW obtained from a stable Markov process taking values in the phase space $\mathbb{R}^{d} \times(0,+\infty)$ has been considered in [20]. It should be noted that, similarly to ibid., the jump distribution of the CTRW (given by $P(x, \mathrm{~d} y)$ ) considered in the present paper is allowed to depend on the current location of the random walker. This type of situation may occur in applications; see the example related to the quantum transport problem presented in Section 4.

When $\left\{X_{n}, n \geq 0\right\}$ is a sequence of i.i.d. random variables, this problem has been investigated in [2]. In [22] the result is generalized to the case of triangular arrays with rowwise independent random variables. From Theorem 3.1 of [2] and Theorem 2.1 of [22], it follows in particular that if $\left\{\left(N^{-1 / \beta} S_{[N t]}, N^{-1 / \alpha} t_{[N t]}\right), t \geq 0\right\}$ converges in law over $D\left([0,+\infty) ; \mathbb{R}^{2}\right)$, with the $J_{1}$ topology, to a Lévy process $\left\{\left(S_{t}, T_{t}\right), t \geq 0\right\}$, whose components have no common jumps, then $\left\{N^{-\alpha / \beta} W(N t), t \geq 0\right\}$ converges in law over $D[0,+\infty)$ with the $M_{1}$ topology to

$$
\zeta_{s}:=S_{T_{s}^{-1}}, \quad s \geq 0
$$

where $T_{s}^{-1}:=\inf \left[t: T_{t}>s\right]$ (the first passage time) is the right inverse of the $\alpha$-stable subordinator $\left\{T_{t}, t \geq 0\right\}$.

When common jumps of the components of $\left\{\left(S_{t}, T_{t}\right), t \geq 0\right\}$ are admitted with positive probability, the situation is more complex and only some partial results concerning convergence are available. In Theorem 3.4 of [2], with the correction to the statement of the result given in [3], it was shown that one-dimensional statistics of $N^{-\alpha / \beta} W(N t)$ weakly converge to the law of $\zeta_{t}^{-}$(the definition of the process is given in (2.19)).

In this paper we formulate sufficient conditions for a Markov chain $\left\{X_{n}, n \geq 0\right\}$, see Theorems 2.4 and 2.5 below, that guarantee the convergence in law of $\left\{N^{-\alpha / \beta} W(N t), t \geq 0\right\}$. As for the hypotheses made about the Markov chain, we assume that the measure $\pi$ satisfies the spectral gap estimate; see Condition 2.1. Moreover, the transition probabilities satisfy some additional regularity assumptions; see Conditions 2.2 and 2.4. It was shown in [18] that, under such conditions, both $\left\{N^{-1 / \beta} S_{[N t]}, t \geq 0\right\}$ and $\left\{N^{-1 / \alpha} t_{[N t]}, t \geq 0\right\}$ converge in law over $D\left([0,+\infty)\right.$, with the $J_{1}$ topology, to respective Lévy processes. We strengthen this result and obtain the joint convergence in law of the two-dimensional processes $\left\{\left(N^{-1 / \beta} S_{[N t]}\right.\right.$, $\left.\left.N^{-1 / \alpha} t_{[N t]}\right), t \geq 0\right\}$ to a respective Lévy process; see Theorems 2.1-2.3. We give a sufficient condition, formulated in terms of the joint law of $(V(x), \tau(x))$ under $\pi$ (see (2.14)), which 
precludes the possibility of jumps of the limiting Lévy process occurring simultaneously with positive probability. The above plus an argument from [2] yield the convergence of $\left\{N^{-\alpha / \beta} W(N t), t \geq 0\right\}$. Furthermore, we prove that when the joint law of $(V(x), \tau(x))$ under $\pi$ is such that the jumps of the limiting Lévy process have to occur simultaneously almost surely (a.s.), see condition (2.16), then the convergence of the CTRW still holds. This is achieved by a careful analysis of the convergence of the right inverses of $\left\{N^{-1 / \alpha} t_{[N t]}, t \geq 0\right\}$. We prove that the convergence of these processes holds in a sense that allows us to control the size of the respective plateaus; see Lemma 3.2. This, in turn, suffices to prove the convergence of the relevant CTRW. The limiting process is $\left\{\zeta_{s}, s \geq 0\right\}$, when no common jumps are allowed for the limit $\left\{\left(S_{t}, T_{t}\right), t \geq 0\right\}$ (see Theorem 2.4), or $\left\{\zeta_{s}^{-}:=S_{T_{s}^{-1}-}, s \geq 0\right\}$ (see Theorem 2.5; the process is defined in (2.19)). This result is in agreement with the results obtained in the i.i.d. case in [19] and [28].

Having in mind possible applications we formulate the result for a counterpart of the CTRW that arises when $\left\{N^{-\alpha / \beta} S(N t), t \geq 0\right\}$ is replaced by a process obtained by linear interpolation of its nodal points. Finally, we apply our results to describe the limiting behavior of a jump process $\left\{K_{s}, s \geq 0\right\}$ on a one-dimensional torus that arises in quantum transport theory; see (4.1). This process is the projection onto a 0 -fiber of the solution of a translation invariant Lindblad equation. It possesses a unique $\sigma$-finite invariant measure, absolutely continuous with respect to the Lebesgue measure; see Proposition 4.1. The dynamics of the process are completely mixing and its one-dimensional statistics converge to a mixture of delta-type measures supported on the set $[\tau=+\infty]$; see Theorem 4.1. As an application of Theorem 2.4, we also obtain (see Corollary 4.1) the convergence in law of additive functionals of the type $N^{-\alpha / \beta} \int_{0}^{N t} V_{0}\left(K_{s}\right) \mathrm{d} s$. In the particular case considered in [9] the torus is the interval [ $\left.-\pi, \pi\right]$ whose endpoints are identified, $\tau(-k)=\tau(k)$ and $\tau(k) \sim|k \pm \pi / 2|^{-2}$, as $|k \pm \pi / 2| \ll 1$, so $\alpha=\frac{1}{2}$. We assume that $V_{0}$ is odd, i.e. $V_{0}(-k)=-V_{0}(k)$. In addition, we suppose that either $V_{0}(k) \sim|k \pm \pi / 2|$, as $|k \pm \pi / 2| \ll 1$, and $V_{0}$ is bounded otherwise, or $V_{0}(k) \sim|k \pm \pi / 2|^{\gamma}$, as $|k \pm \pi / 2| \ll 1$ for some $\gamma>1$, and $V_{0}(k) \sim\left|k \pm k_{0}\right|^{-1}$ for some $k_{0} \notin\{-\pi / 2, \pi / 2\}$. The law of $V(k, \tau):=V_{0}(k) \tau$ belongs then to the normal domain of attraction of the Cauchy law, so $\beta=\frac{1}{2}$. We conclude therefore that the scaling properties of the limiting process are the same as those of the Brownian motion. We call such a process a fake diffusion.

\section{Preliminaries and statements of the main results}

\subsection{A Markov chain}

Let $(E, d)$ be a Polish metric space, and let $\&$ be its Borel $\sigma$-algebra. Assume that $\left\{X_{n}, n \geq\right.$ $0\}$ is a Markov chain with state space $E$ and that $\pi$, the law of $X_{0}$, is invariant and ergodic for the chain. We suppose that the following hypotheses are satisfied.

Condition 2.1. Spectral gap condition:

$$
\sup \left[\|P f\|_{L^{2}(\pi)}: f \perp 1,\|f\|_{L^{2}(\pi)}=1\right]=a<1 .
$$

Since $P$ is also a contraction in $L^{1}(\pi)$ and $L^{\infty}(\pi)$, we conclude, via the Riesz-Thorin interpolation theorem, that, for any $p \in[1,+\infty)$,

$$
\|P f\|_{L^{p}(\pi)} \leq a^{1-|2 / p-1|}\|f\|_{L^{p}(\pi)}
$$

for all $f \in L^{p}(\pi)$ such that $\int f \mathrm{~d} \pi=0$.

We also suppose that the absolute continuous part of the transition probability function has some regularity property. Namely, we assume that the following condition holds. 
Condition 2.2. There exist a measurable family of Borel measures $Q(x, \mathrm{~d} y)$ and a measurable, nonnegative function $p(x, y)$ such that

$$
P(x, \mathrm{~d} y)=P_{a}(x, \mathrm{~d} y)+Q(x, \mathrm{~d} y) \quad \text { for all } x \in E,
$$

where $P_{a}(x, \mathrm{~d} y):=p(x, y) \pi(\mathrm{d} y)$ and

$$
C(2):=\sup _{y \in E} \int p^{2}(x, y) \pi(\mathrm{d} x)<+\infty .
$$

A simple consequence of (2.3) and the fact that $\pi$ is invariant is that

$$
\int p(x, y) \pi(\mathrm{d} y) \leq 1 \text { and } \int p(y, x) \pi(\mathrm{d} y) \leq 1 \quad \text { for all } x \in E .
$$

A consequence of condition (2.4) is that $P$ extends to a bounded operator from $L^{1}(\pi)$ to $L^{2}(\pi)$.

\subsection{The renewal process}

Suppose that $\tau: E \rightarrow[0,+\infty)$ is measurable over $(E, \mathscr{E})$ and satisfies the following condition.

Condition 2.3. There exist $\alpha \in(0,1)$ and $c_{\alpha}>0$ such that

$$
\lim _{\lambda \rightarrow+\infty} \lambda^{\alpha} \pi(\tau \geq \lambda)=c_{\alpha},
$$

and there exists $t_{*}>0$ such that $\tau(x) \geq t_{*}>0$ for all $x \in E$.

We assume that Condition 2.3 holds in order to avoid the issue of explosions or accumulation points. Furthermore, we suppose that the tails of $\tau$ under the singular part are controlled by those corresponding to the absolutely continuous part uniformly with respect to the initial state, that is, we suppose that the following condition holds.

Condition 2.4.

$$
\sup _{\lambda \geq 0, x \in E} \frac{Q(x,[\tau \geq \lambda])}{P_{a}(x,[\tau \geq \lambda])}<+\infty .
$$

Let $\left\{X_{n}, n \geq 0\right\}$ be a Markov chain as in the previous section, and let $t_{0}:=0$ and

$$
t_{N}:=\sum_{k=0}^{N-1} \tau\left(X_{k}\right) \quad \text { for } N \geq 1 .
$$

For a given $t>0$, define $n(t)$ as the unique (random) integer that satisfies the condition

$$
t \in\left[t_{n(t)}, t_{n(t)+1}\right) .
$$

\subsection{An observable and the CRTW process}

Suppose now that $V: E \rightarrow \mathbb{R}$ is measurable. Let $S_{0}:=0$ and

$$
S_{N}:=\sum_{k=0}^{N-1} V\left(X_{k}\right) \quad \text { for } N \geq 1
$$


We will assume that either

$$
V \in L^{2}(\pi) \text { and } \int V \mathrm{~d} \pi=0
$$

or, in case $V$ does not belong to $L^{2}(\pi)$, that there exist $\beta \in(0,2)$ and two nonnegative constants $c_{\beta}^{+}$and $c_{\beta}^{-}$satisfying $c_{\beta}^{+}+c_{\beta}^{-}>0$ and

$$
\pi(V \geq \lambda)=\frac{c_{\beta}^{+}}{\lambda^{\beta}}(1+o(1)), \quad \pi(V \leq-\lambda)=\frac{c_{\beta}^{-}}{\lambda^{\beta}}(1+o(1)), \quad \text { as } \lambda \rightarrow+\infty .
$$

Furthermore, $V$ is supposed to be centered when $\beta \in(1,2)$. In analogy to condition (2.6), we assume that the tails of $V$ under the singular part of $P(x, \cdot)$ are controlled by those of the absolutely continuous part, i.e.

$$
\sup _{\lambda \geq 0, x \in E} \frac{Q(x,[|V| \geq \lambda])}{P_{a}(x,[|V| \geq \lambda])}<+\infty .
$$

We define the CTRW process $W(t):=S_{n(t)}, t \geq 0$. Its trajectories belong to the space of càdlàg functions $D[0,+\infty)$; we will abbreviate this space by $\mathscr{D}$ in what follows. We define the piecewise-linear counterpart of the CTRW by

$$
\hat{W}(t):=S_{n(t)}+\frac{t-t_{n(t)}}{t_{n(t)+1}-t_{n(t)}} V\left(X_{n(t)}\right) \quad \text { for } t \in\left[t_{n(t)}, t_{n(t)+1}\right) .
$$

In our subsequent notation we write $\mathcal{C}:=C[0,+\infty)$.

\subsection{Convergence to a Lévy process}

The results presented in this section extend those of [18] to the case of two-dimensional Markov chains. They can be proved using quite similar arguments. For the convenience of the reader, we present the main points of the respective proofs in Appendices A and B.

Suppose that the hypotheses made in Sections 2.1-2.2 hold and that $K_{N}$ is an increasing sequence converging to $\infty$. Our immediate concern is the question of the convergence of the joint processes $\left\{\left(S_{t}^{(N)}, T_{t}^{(N)}\right), t \geq 0\right\}$ as $N \rightarrow+\infty$. Here, when $\beta \neq 1$,

$$
T_{t}^{(N)}:=K_{N}^{-1 / \alpha} t_{\left[K_{N} t\right]}, \quad S_{t}^{(N)}:=K_{N}^{-1 / \beta} S_{\left[K_{N} t\right]},
$$

and when $\beta=1$,

$$
S_{t}^{(N)}:=K_{N}^{-1} S_{\left[K_{N} t\right]}-v_{N} t
$$

where $v_{N}:=\int V \mathbf{1}_{\left\{|V| \leq K_{N}\right\}} \mathrm{d} \pi$. Let

$$
\psi_{\beta}(\xi):=\int_{\mathbb{R}} e_{\beta}(\xi, \lambda) v_{\beta}(\mathrm{d} \lambda)
$$

where

$$
e_{\beta}(\xi, \lambda):= \begin{cases}\mathrm{e}^{i \lambda \xi}-1, & \beta \in(0,1), \\ \mathrm{e}^{i \xi \lambda}-1-i \xi \lambda \mathbf{1}_{[-1,1]}(\lambda), & \beta=1, \\ \mathrm{e}^{i \lambda \xi}-1-i \lambda \xi, & \beta \in(1,2),\end{cases}
$$


and $v_{\beta}(\mathrm{d} \lambda):=\beta c_{\beta}(\lambda)|\lambda|^{-1-\beta} \mathrm{d} \lambda$. Here $c_{\beta}(\lambda)$ equals $c_{\beta}^{+}$for $\lambda>0$ and $c_{\beta}^{-}$for $\lambda<0$. Consider a Lévy process $\left\{\left(S_{t}, T_{t}\right), t \geq 0\right\}$ given by $\mathrm{Ee}^{i \xi_{1} S_{t}+i \xi_{2} T_{t}}=\mathrm{e}^{t \psi\left(\xi_{1}, \xi_{2}\right)}$, where

$$
\begin{aligned}
\psi\left(\xi_{1}, \xi_{2}\right) & :=\psi_{\beta}\left(\xi_{1}\right)+\psi_{\alpha}\left(\xi_{2}\right) \\
& =\int e_{\alpha, \beta}\left(\xi_{1}, \xi_{2}, \lambda_{1}, \lambda_{2}\right) \nu_{*}\left(\mathrm{~d} \lambda_{1}, \mathrm{~d} \lambda_{2}\right), \quad\left(\xi_{1}, \xi_{2}\right) \in \mathbb{R}^{2}
\end{aligned}
$$

where the coefficients $c_{\alpha}^{+}$and $c_{\alpha}^{-}$appearing in the definition of $\psi_{\alpha}(\cdot)$ are equal then to $c_{\alpha}$ (see (2.5)) and 0 , respectively,

$$
e_{\alpha, \beta}\left(\xi_{1}, \xi_{2}, \lambda_{1}, \lambda_{2}\right):= \begin{cases}\mathrm{e}^{i\left(\lambda_{1} \xi_{1}+\lambda_{2} \xi_{2}\right)}-1, & \beta \in(0,1), \\ \mathrm{e}^{i\left(\lambda_{1} \xi_{1}+\lambda_{2} \xi_{2}\right)}-1-i \xi_{1} \lambda_{1} \mathbf{1}_{[-1,1]}\left(\lambda_{1}\right), & \beta=1, \\ \mathrm{e}^{i\left(\lambda_{1} \xi_{1}+\lambda_{2} \xi_{2}\right)}-1-i \lambda_{1} \xi_{1}, & \beta \in(1,2),\end{cases}
$$

and

$$
\nu_{*}\left(\mathrm{~d} \lambda_{1}, \mathrm{~d} \lambda_{2}\right):=v_{\beta}\left(\mathrm{d} \lambda_{1}\right) \delta_{0}\left(\mathrm{~d} \lambda_{2}\right)+\delta_{0}\left(\mathrm{~d} \lambda_{1}\right) v_{\alpha}\left(\mathrm{d} \lambda_{2}\right) .
$$

In our first result we adopt the hypothesis that $\tau(x)$ and $|V(x)|$ cannot be large together. Namely, we assume that

$$
\pi[\tau \geq \lambda,|V| \geq \lambda] \leq \frac{C_{*}}{\lambda \gamma}, \quad \lambda>0,
$$

for some $C_{*}>0$ and $\gamma>\alpha \vee \beta$.

Theorem 2.1. Suppose that the assumptions made in (2.9) and (2.14) hold, and that $V$ is centered when $\beta \in(1,2)$. Then the following statements hold.

(i) If $\beta \neq 1$ then the joint laws of $\left\{\left(S_{t}^{(N)}, T_{t}^{(N)}\right), t \geq 0\right\}$ converge in law as $N \rightarrow+\infty$ to $\left\{\left(S_{t}, T_{t}\right), t \geq 0\right\}$ on $D_{2}:=D\left([0,+\infty), \mathbb{R}^{2}\right)$ with the $J_{1}$ topology.

(ii) If $\beta=1$, we assume that, for some $\beta^{\prime}>1$,

$$
\sup _{N \geq 1}\left\|P V_{N}\right\|_{L^{\beta^{\prime}}(\pi)}<+\infty
$$

where $V_{N}:=V \mathbf{1}_{\left\{|V| \leq K_{N}\right\}}$. Then the statement of part (i) also holds in this case where $S_{t}^{(N)}$ is given by $(2.11)$.

In our next result we allow the jumps of the components of $\left\{\left(S_{t}^{(N)}, T_{t}^{(N)}\right), t \geq 0\right\}$ to occur at the same time. More specifically, let

$$
\rho(\lambda):=C_{\alpha, \beta}|\lambda|^{\beta / \alpha}, \quad \lambda \in \mathbb{R},
$$

where $C_{\alpha, \beta}:=c_{\alpha}\left(c_{\beta}^{-}+c_{\beta}^{+}\right)^{-1}$. Suppose that, for some $C_{*}>0$ and $\gamma>\alpha$, we have

$$
\pi[|\tau-\rho \circ V| \geq \lambda] \leq \frac{C_{*}}{\lambda \gamma} \quad \text { for all } \lambda>0 .
$$

Now consider a Lévy process $\left\{\left(S_{t}, T_{t}\right), t \geq 0\right\}$ such that

$$
\psi\left(\xi_{1}, \xi_{2}\right):=\int_{\mathbb{R}^{2}} e_{\alpha, \beta}\left(\xi_{1}, \xi_{2}, \lambda_{1}, \lambda_{2}\right) \nu_{*}\left(\mathrm{~d} \lambda_{1}, \mathrm{~d} \lambda_{2}\right),
$$

where $e_{\alpha, \beta}\left(\xi_{1}, \xi_{2}, \lambda_{1}, \lambda_{2}\right)$ is given by (2.12) and

$$
v_{*}\left(\mathrm{~d} \lambda_{1}, \mathrm{~d} \lambda_{2}\right):=\delta_{0}\left(\lambda_{2}-\rho\left(\lambda_{1}\right)\right) v_{\beta}\left(\mathrm{d} \lambda_{1}\right) \mathrm{d} \lambda_{2} .
$$


Theorem 2.2. Suppose that (2.16) holds. Then the convergence statements analogous to Theorem 2.1(i) and (ii) still hold. The only difference is that the limiting Lévy process is described by the exponent given in (2.17).

Finally, when $V \in L^{2}(\pi)$, i.e. (2.8) holds, we have the following result.

Theorem 2.3. The laws of $\left\{\left(S_{t}^{(N)}, T_{t}^{(N)}\right), t \geq 0\right\}$ converge as $N \rightarrow+\infty$ over $\mathscr{D} \times \mathscr{D}$ with the product of the uniform and $J_{1}$ topologies to the joint law of independent Lévy processes: $\left\{\left(S_{t}, T_{t}\right), t \geq 0\right\}$. The first component is a zero-mean Brownian motion and the Lévy exponent of the second component equals $\psi_{\alpha}(\xi)$.

\subsection{Convergence of CTRWs}

Our first result, concerning the convergence of the CTRW, is as follows.

Theorem 2.4. Under the assumptions of either Theorem 2.1 or Theorem 2.3, the processes $\left\{N^{-\alpha / \beta} W(N t), t \geq 0\right\}$ converge in law in the $M_{1}$ topology of $\mathcal{D}$ as $N \rightarrow+\infty$ to $\left\{\zeta_{t}:=\right.$ $\left.S_{s(t)}, t \geq 0\right\}$, where $\left\{\left(S_{t}, T_{t}\right), t \geq 0\right\}$ is an appropriate Lévy process and $\{s(t), t \geq 0\}$ is the right inverse of $\left\{T_{t}, t \geq 0\right\}$. The result also holds when the process $W(t)$ is replaced by the linear interpolation process $\hat{W}(t)$. In the latter case, under the assumptions of Theorem 2.3, the convergence in law holds over $\mathcal{C}$.

Remark 2.1. If $\left\{\left(S_{t}, T_{t}\right), t \geq 0\right\}$ is such that its first component is a Brownian motion then the components of the process are independent; see Theorem 2.3. In that case $\left\{\zeta_{s}=S_{T_{s}^{-1}}, s \geq 0\right\}$ is called a Mittag-Leffler process. It is non-Markovian and arises as a limit of an appropriately scaled additive functional of a Markov process, whose resolvent, applied at the observable, obeys the power law at the bottom of the spectrum of the generator; see [1] and [10]. We refer the reader to, e.g. [16] and the references therein for an extensive review of the results concerning this particular case.

Given the Lévy process $\left\{\left(S_{t}, T_{t}\right), t \geq 0\right\}$ as described in Theorem 2.2, define

$$
\zeta_{t}^{-}=\lim _{N \rightarrow+\infty} S_{s(t)-1 / N}, \quad t \geq 0 .
$$

The limit is understood a.s. in the $J_{1}$ topology of $\mathscr{D}$. Here $S_{t}=0$ when $t<0$. Observe that although the notation suggests otherwise, the process $\left\{\zeta_{t}^{-}, t \geq 0\right\}$ is càdlàg, as a limit of càdlàg processes in the $J_{1}$ topology.

Theorem 2.5. Under the assumptions of Theorem 2.2, the processes $\left\{N^{-\alpha / \beta} W(N t), t \geq 0\right\}$ converge in law in the $J_{1}$ topology of $\mathscr{D}$ as $N \rightarrow+\infty$ to $\left\{\zeta_{t}^{-}, t \geq 0\right\}$, defined above.

Remark 2.2. We point out here that the limiting processes described in Theorems 2.4 and 2.5 have a scale invariance property. Namely, the laws of $\left\{\zeta_{a t}, t \geq 0\right\}$ and that of $\left\{a^{\alpha / \beta} \zeta_{t}, t \geq 0\right\}$ are identical for each $a>0$. The same scaling invariance also applies to the process $\left\{\zeta_{t}^{-}, t \geq 0\right\}$. This remark follows easily from the fact that, in the cases considered in both theorems, the Lévy processes $\left\{\left(S_{t a^{\alpha}}, T_{t a^{\alpha}}\right), t \geq 0\right\}$ and $\left\{\left(a^{\alpha / \beta} S_{t}, a T_{t}\right), t \geq 0\right\}$ have identical Lévy exponents. Thus, the joint laws of $\left\{\left(S_{t a^{\alpha}}, T_{t a^{\alpha}}\right), t \geq 0\right\}$ and those of $\left\{\left(a^{\alpha / \beta} S_{t}, a T_{t}\right), t \geq 0\right\}$ over $\mathscr{D}_{2}$ are identical. This in turn easily implies the scale invariance property. 


\section{The proofs of Theorems 2.4 and 2.5}

\subsection{The case when jumps cannot occur together}

Here we assume that the sets of discontinuity points for the components of the limiting processes $\left\{S_{t}, t \geq 0\right\}$ and $\left\{T_{t}, t \geq 0\right\}$ are a.s. disjoint, i.e. that either the assumptions of Theorem 2.1 or 2.3 hold. Then the weak convergence of $\left\{N^{-\alpha / \beta} W(N t), t \geq 0\right\}$ can be proven in exactly the same way as in Theorem 3.1 of [2]. We only show the convergence of the linear interpolation process $N^{-\alpha / \beta} \hat{W}(N t)$.

Assume first that the assumptions of Theorem 2.1 hold. For a given $t>0$, recall that $n(N t)$ is the (random) integer given by (2.7). Let $K_{N}:=N^{\alpha}$. We define

$$
S_{t}^{(N)}:=K_{N}^{-1 / \beta} \sum_{n=0}^{\left[K_{N} t\right]-1} V\left(X_{n}\right), \quad T_{t}^{(N)}:=K_{N}^{-1 / \alpha} t_{\left[K_{N} t\right]},
$$

$s_{N}(t)$ to be the right-continuous inverse of $\left\{T_{t}^{(N)}, t \geq 0\right\}$, i.e. $s_{N}(t):=\inf \left[u: T_{u}^{(N)}>t\right]$, and $s_{N}^{*}(t):=s_{N}(t)-N^{-\alpha}=\max \left[u: T_{u}^{(N)} \leq t\right]$. Denote by $\hat{S}_{t}^{(N)}$ the process whose paths are obtained by the linear interpolation between the points $\left(m K_{N}^{-1}, S_{m K_{N}^{-1}}\right)$, where $m \geq 0$ is an integer. Then $N^{-\alpha / \beta} \hat{W}(N t)=\hat{S}_{S_{N}^{*}(t)}^{(N)}$. The following result allows us to replace the first coordinate process in the statement of Theorem 2.1 by its linear interpolation.

Lemma 3.1. Under the assumptions of Theorem 2.1, the processes $\left\{\left(\hat{S}_{t}^{(N)}, T_{t}^{(N)}\right), t \geq 0\right\}$ converge in law as $N \rightarrow+\infty$ over $\mathscr{D} \times \mathscr{D}$ with the product of the $M_{1}$ topologies to the Lévy process $\left\{\left(S_{t}, T_{t}\right), t \geq 0\right\}$, as in the statement of Theorem 2.1.

Before proving the lemma we show how to use it to complete the proof of Theorem 2.4. By Skorokhod's embedding theorem we define a family of processes $\left\{\left(U_{t}^{(N)}, V_{t}^{(N)}\right), t \geq 0\right\}$ such that

1. the law of $\left\{\left(U_{t}^{(N)}, V_{t}^{(N)}\right), t \geq 0\right\}$ is identical to that of $\left\{\left(\hat{S}_{t}^{(N)}, T_{t}^{(N)}\right), t \geq 0\right\}$ for each $N \geq 1$,

2. $\left\{\left(U_{t}^{(N)}, V_{t}^{(N)}\right), t \geq 0\right\}$ converges a.s., in $\mathscr{D} \times \mathcal{D}$ equipped with the product $M_{1}$ topology, to $\left\{\left(S_{t}, T_{t}\right), t \geq 0\right\}$. Here the limiting process is as in Theorem 2.1.

Suppose that $\left\{u_{N}(t), t \geq 0\right\}$ is the right inverse of $\left\{V_{t}^{(N)}, t \geq 0\right\}$ and that $u_{N}^{*}(t):=u_{N}(t)-$ $1 / N^{\alpha}$. The law of $\left\{Y_{t}^{(N)}:=U_{u_{N}^{*}(t)}^{(N)}, t \geq 0\right\}$ coincides with that of $N^{-\alpha / \beta} \hat{W}(N t)$. Moreover, both $\left\{U_{t}^{(N)}, t \geq 0\right\}$ and $\left\{u_{N}^{*}(t), t \geq 0\right\}$ converge a.s. as $N \rightarrow+\infty$ in the $M_{1}$ topology to $\left\{S_{t}, t \geq 0\right\}$ and $\{s(t), t \geq 0\}$ (the right inverse of $\left\{T_{t}, t \geq 0\right\}$ ), respectively. In fact, since $\{s(t), t \geq 0\}$ is a.s. continuous, the latter sequence converges in the uniform topology. Theorem 13.2.4 of [29] therefore implies that the processes $\left\{Y_{t}^{(N)}, t \geq 0\right\}$ converge in the $M_{1}$ topology to $\left\{Y_{t}, t \geq 0\right\}$ a.s., provided that the sets of discontinuities of $\left\{S_{t}, t \geq 0\right\}$ and $\left\{T_{t}, t \geq 0\right\}$ are a.s. disjoint. This however is a simple consequence of the independence of these processes.

Proof of Lemma 3.1. Suppose that $T>0$. Recall how the $M_{1}$ topology on $D[0, T]$ can be metrized; see [29, p. 476] for details. For a given $X \in D[0, T]$, we define by $\Gamma_{X}$ the graph of $X$, i.e. the subset of $\mathbb{R}^{2}$ given by

$$
\Gamma_{X}:=[(t, z): t \in[0, T], z=c X(t-)+(1-c) X(t) \text { for some } c \in[0,1]] .
$$


On $\Gamma_{X}$ we define an order by letting $\left(t_{1}, z_{1}\right) \leq\left(t_{2}, z_{2}\right)$ if and only if $t_{1}<t_{2}$, or $t_{1}=t_{2}$ and $\left|X\left(t_{1}-\right)-z_{1}\right| \leq\left|X\left(t_{1}-\right)-z_{2}\right|$. Denote by $\Pi(X)$ the set of all continuous mappings $\gamma=\left(\gamma^{(1)}, \gamma^{(2)}\right):[0,1] \rightarrow \Gamma_{X}$ that are nondecreasing, i.e. $t_{1} \leq t_{2}$ implies that $\gamma\left(t_{1}\right) \leq \gamma\left(t_{2}\right)$. The metric $d(\cdot, \cdot)$ is defined as follows:

$$
d\left(X_{1}, X_{2}\right):=\inf \left[\left\|\gamma_{1}^{(1)}-\gamma_{2}^{(1)}\right\|_{\infty} \vee\left\|\gamma_{1}^{(2)}-\gamma_{2}^{(2)}\right\|_{\infty}, \gamma_{i}=\left(\gamma_{i}^{(1)}, \gamma_{i}^{(2)}\right) \in \Pi\left(X_{i}\right), i=1,2\right]
$$

This metric provides a metrization of the $M_{1}$ topology; see [29, Theorem 13.2.1].

For any $\gamma_{1} \in \Gamma_{S^{(N)}}$, we define $\gamma_{2} \in \Gamma_{\hat{S}^{(N)}}$ as follows. Suppose that $\gamma_{1}(t)$ belongs to the graph corresponding to $\left(t, S_{t}^{(N)}\right), t \in\left[m K_{N}^{-1},(m+1) K_{N}^{-1}\right)$ for an integer $m \geq 0$. Let $\gamma_{2}(t)$ be the nearest neighbor projection of $\gamma_{1}(t)$ onto the segment joining $\left(m K_{N}^{-1}, S_{m K_{N}^{-1}}^{(N)}\right)$ with $\left((m+1) K_{N}^{-1}, S_{(m+1) K_{N}^{-1}-}^{(N)}\right)$. We can use these two parametrizations to estimate the distance $d$ :

$$
d\left(S_{.}^{(N)}, \hat{S}_{.}^{(N)}\right) \leq C K_{N}^{-1 / 2(1+1 / \beta)} \max _{0 \leq n \leq\left[T K_{N}\right]}\left|V\left(X_{n}\right)\right|^{1 / 2}
$$

for some deterministic constant $C>0$ independent of $N$. Hence, for any $\eta>0$, we obtain

$$
\mathrm{P}\left[d\left(S^{(N)}, \hat{S}_{.}^{(N)}\right) \geq \eta\right] \leq C K_{N}^{-\beta} \rightarrow 0
$$

as $N \rightarrow+\infty$. The lemma is then a consequence of (3.1) and Theorem 2.1.

When, on the other hand, the assumptions of Theorem 2.3 hold, we can conclude that, for each $T, \eta>0$,

$$
\lim _{N \rightarrow+\infty} \mathrm{P}\left[\sup _{t \in[0, T]}\left|S_{t}^{(N)}-\hat{S}_{t}^{(N)}\right| \geq \eta\right]=0,
$$

which implies the weak convergence of the linear interpolation process $\left\{N^{-\alpha / \beta} \hat{W}(N t), t \geq 0\right\}$ over $\mathcal{C}$ to a Mittag-Leffler process $\left\{\zeta_{t}, t \geq 0\right\}$. To show (3.2), note that the expression under the limit on the left-hand side can be estimated from above by

$$
\begin{aligned}
\left.\mathrm{P} \max _{0 \leq n \leq K_{N} T}\left|V\left(X_{n}\right)\right| \geq K_{N}^{1 / 2} \eta\right] & \leq K_{N} T \pi\left(|V| \geq K_{N}^{1 / 2} \eta\right) \\
& \leq \frac{K_{N} T}{\left(\eta K_{N}^{1 / 2}\right)^{2}} \int_{\left[|V| \geq K_{N}^{1 / 2} \eta\right]} V^{2} \mathrm{~d} \pi \\
& =\frac{T}{\eta^{2}} \int_{\left[|V| \geq K_{N}^{1 / 2} \eta\right]} V^{2} \mathrm{~d} \pi \\
& \rightarrow 0
\end{aligned}
$$

as $N \rightarrow+\infty$.

\subsection{The case when jumps occur together}

We assume that the hypotheses of Theorem 2.2 hold and admit $K_{N}:=N^{\alpha}$, as in the previous subsection. Using Skorokhod's embedding theorem, define a family of processes $\left\{\left(U_{t}^{(N)}, V_{t}^{(N)}\right), t \geq 0\right\}$ such that

1. the law of $\left\{\left(U_{t}^{(N)}, V_{t}^{(N)}\right), t \geq 0\right\}$ coincides with that of $\left\{\left(S_{t}^{(N)}, T_{t}^{(N)}\right), t \geq 0\right\}$ for each $N \geq 1$, 
2. $\left\{\left(U_{t}^{(N)}, V_{t}^{(N)}\right), t \geq 0\right\}$ converges a.s. in the $J_{1}$ topology of $\mathscr{D}_{2}$ to $\left\{\left(S_{t}, T_{t}\right), t \geq 0\right\}$. The latter process is as in the statement of Theorem 2.2. The above means that, for any $L>0$, we can find a sequence $\left\{\lambda_{n}, n \geq 1\right\}$ of increasing homeomorphisms in $[0, L]$ such that $\lambda_{N}(0)=0$ and $\lambda_{N}(L)=L$, and

$$
\begin{gathered}
\sup _{t \in[0, L]}\left|\lambda_{N}(t)-t\right| \rightarrow 0 \\
\sup _{t \in[0, L]}\left|U_{\lambda_{N}(t)}^{(N)}-S_{t}\right| \rightarrow 0, \quad \text { and } \sup _{t \in[0, L]}\left|V_{\lambda_{N}(t)}^{(N)}-T_{t}\right| \rightarrow 0
\end{gathered}
$$

as $N \rightarrow+\infty$.

Let $u_{N}(t)$ and $s(t)$ be the respective right inverses of $V_{t}^{(N)}$ and $T_{t}$. On the other hand, if $u_{N}^{*}(t):=$ $\max \left[s: U_{s}^{(N)} \leq t\right]$ then $u_{N}^{*}(t)=u_{N}(t)-1 / N^{\alpha}$. Observe that the CTRW $\left\{N^{\alpha / \beta} W(N t), t \geq\right.$ $0\}$ has the same law as $\left\{U_{u_{N}^{*}(t)}^{(N)}, t \geq 0\right\}$.

We show the following.

Theorem 3.1. Under the assumptions of Theorem 2.2, the processes $\left\{U_{u_{N}^{*}(t)}^{(N)}, t \geq 0\right\}$ converge in law over $\mathcal{D}$, with the $J_{1}$ topology, as $N \rightarrow+\infty$ to $\left\{\zeta_{t}^{-}, t \geq 0\right\}$ defined in (2.19).

Proof. The proof relies on a careful analysis of convergence of processes $u_{N}(\cdot)$ to $s(\cdot)$. We know that they converge uniformly on any compact interval. In fact, as we show in Lemma 3.2 below for $t$ in a plateau of $s(\cdot)$ of a fixed size, we have $\lambda_{N}^{-1} \circ u_{N}(t)=s(t)$ for a sufficiently large $N$. Matching plateaus of $s(\cdot)$ with those of $\lambda_{N}^{-1} \circ u_{N}(\cdot)$ we define homeomorphisms $\Lambda_{N}$ such that $\lim _{N \rightarrow+\infty} U_{u_{N} \circ \Lambda_{N}(t)}^{(N)}=S_{S(t)}$ uniformly on the set of plateaus of $s(\cdot)$ of a fixed size. To show that this convergence also extends to the entire $\left[0, T_{L}\right]$, we use the fact that the plateaus of $s(\cdot)$ also correspond to the jumps of $\left\{S_{t}, t \geq 0\right\}$; see Lemma 3.3. This is due to the fact that jumps of $S_{t}$ and $T_{t}$ are matched by function $\rho(\cdot)$. Therefore, outside the large size plateaus of $s(\cdot)$ the trajectory $\left\{S_{s(t)}, t \geq 0\right\}$ cannot suffer large jumps. Since $\left|u_{N} \circ \Lambda_{N}(t)-s(t)\right|$ is small for sufficiently large $N$, we can easily conclude that $\left|U_{u_{N} \circ \Lambda_{N}}^{(N)}(t)-S_{s(t)}\right|$ is also small. This fact also implies that $\lim _{N \rightarrow+\infty}\left|U_{u_{N}^{*} \circ \Lambda_{N}}^{(N)}(t)-S_{S(t)-1 / N^{\alpha}}\right|=0$ and the theorem follows.

To provide a rigorous proof of Theorem 3.1, we need some auxiliary results. For each $\delta>0$, we denote by $A_{\delta}=A_{\delta}(s(\cdot))$ the set of 'plateau points' of $s(t)$ of size at least $\delta$, i.e.

$t \in A_{\delta}$ if and only if $s(t)$ is constant in the interval $(t-\delta, t+\delta) \cap\left[0, T_{L}\right]$.

Obviously, $A_{\delta} \subset A_{\delta^{\prime}}$ if $\delta^{\prime} \leq \delta$.

Lemma 3.2. For a fixed $L>0$, the sequence $\left\{u_{N}(\cdot), N \geq 1\right\}$ converges to $s(\cdot)$ in the following sense:

(i) $\left\|\lambda_{N}^{-1} \circ u_{N}-s\right\|_{\infty} \rightarrow 0$ a.s. as $N \rightarrow+\infty$, where the supremum norm is taken over $[0, L]$,

(ii) there exists a decreasing sequence $d_{N} \rightarrow 0$ as $N \rightarrow+\infty$ such that $\lambda_{n}^{-1} \circ u_{n}(t)=s(t)$ for $t \in A_{d_{N}}, n \geq N$, and all $N \geq 1$.

Proof. Let

$$
a_{N}:=\max \left[\left\|V^{(N)} \circ \lambda_{N}-T\right\|_{\infty},\left\|U^{(N)} \circ \lambda_{N}-S\right\|_{\infty},\left\|\lambda_{N}-\mathrm{id}\right\|_{\infty},\left\|\lambda_{N}^{-1}-\mathrm{id}\right\|_{\infty}\right]
$$


and

$$
d_{N}:=\sup _{n \geq N}\left[\left\|V^{(n)} \circ \lambda_{n}-T\right\|_{\infty},\left\|U^{(n)} \circ \lambda_{n}-S\right\|_{\infty},\left\|\lambda_{n}-\mathrm{id}\right\|_{\infty},\left\|\lambda_{n}^{-1}-\mathrm{id}\right\|_{\infty}\right] .
$$

Here id is the identity map on $[0, L]$. From (3.3) and (3.4), we have $d_{N} \rightarrow 0$; thus $a_{N} \rightarrow 0$ as $N \rightarrow+\infty$ also. Since each $u_{N}(t)$ is increasing and $s(t)$ is a.s. continuous, in order to prove (i), it is enough to show that, a.s.,

$$
\lim _{N \rightarrow+\infty} u_{N}(t)=s(t) \quad \text { for all } t \geq 0 .
$$

We first prove that the convergence holds a.s. for any fixed $t$. We claim that

$$
u_{N}(t) \leq s\left(t+a_{N}\right)+a_{N} \quad \text { a.s. }
$$

Indeed, let $\delta>0$ and $s_{*}:=s\left(t+a_{N}\right)+a_{N}+\delta$. Then $s\left(t+a_{N}\right)=s_{*}-a_{N}-\delta<\lambda_{N}^{-1}\left(s_{*}\right)$, by definition (3.5). From the definition of the right inverse, $t+a_{N} \leq T_{s\left(t+a_{N}\right)}$. Since $\left\{T_{s}, s \geq 0\right\}$ is a.s. strictly increasing (see Theorem 21.3 of [25, p. 136]),

$$
V_{s_{*}}^{(N)} \geq T_{\lambda_{N}^{-1}\left(s_{*}\right)}-a_{N}>T_{s\left(t+a_{N}\right)}-a_{N} \geq t,
$$

so

$$
u_{N}(t) \leq s_{*}=s\left(t+a_{N}\right)+a_{N}+\delta .
$$

Since $\delta>0$ was arbitrary, (3.6) follows.

Likewise, we prove that $u_{N}(t) \geq s\left(t-a_{N}\right)-a_{N}$ a.s. and, as a result, we conclude that there exists $\mathbb{D}$, a dense subset of $[0,+\infty)$, such that $\lim _{N \rightarrow+\infty} u_{N}(t)=s(t)$ for $t \in \mathbb{D}$ a.s. Since all the functions $u_{N}$ are increasing, the convergence can be easily extrapolated to the entire $[0,+\infty)$. This completes the proof of part (i).

For any $t \in A_{d_{N}}$ and $n \geq N$, we have

$$
\begin{gathered}
T_{s(t)-} \leq t-d_{N} \leq t+d_{N} \leq T_{S(t)} \\
\left|V_{\lambda_{n} \circ s(t)-}^{(n)}-T_{s(t)-}\right| \leq d_{N}, \quad \text { and } \quad\left|V_{\lambda_{n} \circ s(t)}^{(n)}-T_{s(t)}\right| \leq d_{N} .
\end{gathered}
$$

Therefore, $V_{\lambda_{n} \circ s(t)-}^{(n)} \leq t \leq V_{\lambda_{n} \circ s(t)}^{(n)}$, which proves that $u_{n}(t)=\lambda_{n} \circ s(t)$. This completes the proof of part (ii) and, thus, of the lemma.

For each $t \geq 0$, define $\Delta S_{t}=S_{t}-S_{t-}$.

Lemma 3.3. Let $\left\{d_{m}, m \geq 1\right\}$ be as in the statement of Lemma 3.2. Under the assumptions of Theorem 2.2, we have

$$
\lim _{m \rightarrow \infty} \sup _{t \notin s\left(A_{d_{m}}\right)}\left|\Delta S_{t}\right|=0 \quad \text { in probability. }
$$

Proof. Let

$$
B_{m}:=\left[\text { there exists } t \in[0, L]:\left|\Delta S_{t}\right| \geq \rho^{-1}\left(4 d_{m}\right) \text { and } \Delta T_{t} \leq d_{m}\right],
$$

where $\rho(\cdot)$ is given by (2.15). We show that

$$
\mathrm{P}\left[B_{m}\right]=0 \quad \text { for all } m \geq 1 .
$$


Suppose first that $\beta<1$. Consider the jump process $\left\{Z_{t}^{(r)}:=\left(S_{t}^{(r)}, T_{t}^{(r)}\right), t \geq 0\right\}$, corresponding to the jump measure

$$
\nu_{*}^{(r)}\left(\mathrm{d} \lambda_{1}, \mathrm{~d} \lambda_{2}\right):=\mathbf{1}_{B_{r}^{c}(0)}\left(\lambda_{1}, \lambda_{2}\right) \nu_{*}\left(\mathrm{~d} \lambda_{1}, \mathrm{~d} \lambda_{2}\right) .
$$

Let $z:=v_{*}^{(r)}\left(\mathbb{R}^{2}\right)$. This process can be realized as follows: $Z_{t}^{(r)}=Z_{N(t)}^{(r)}$, where $Z_{n}^{(r)}$ is a sum of $n$ independent random variables distributed according to $z^{-1} \nu_{*}^{(r)}\left(\mathrm{d} \lambda_{1}, \mathrm{~d} \lambda_{2}\right)$ and $N(t)$ is an independent of those variables Poisson process with intensity $z$. Since the jumps of $\left\{Z_{t}^{(r)}, t \geq 0\right\}$ are vectors whose coordinates belong to the support of $\nu_{*}^{(r)}\left(\mathrm{d} \lambda_{1}, \mathrm{~d} \lambda_{2}\right)$ (contained in the curve $\{(\lambda, \rho(\lambda)), \lambda>0\})$, we have

$$
\mathrm{P}\left[B_{m}^{(r)}\right]=0
$$

where

$$
\left.B_{m}^{(r)}:=\text { [there exists } t \in[0, L]:\left|\Delta S_{t}^{(r)}\right| \geq \rho^{-1}\left(3 d_{m}\right) \text { and } \Delta T_{t}^{(r)} \leq 2 d_{m}\right] .
$$

Let $\left\{Z_{t}:=\left(S_{t}, T_{t}\right), t \geq 0\right\}$. It is well known (see, e.g. [7, Theorem 14.27]) that

$$
\lim _{r \rightarrow 0+} \sup _{t \in[0, L]}\left|Z_{t}^{(r)}-Z_{t}\right|=0 \quad \text { in probability. }
$$

Combining (3.9) and (3.10) we obtain (3.8). Thus, (3.7) follows.

The case when $\beta \in[1,2)$ can be concluded similarly. However, then the approximating processes should be of the form $\left\{Z_{t}^{(r)}-c^{(r)} t, t \geq 0\right\}$ for some $c^{(r)}=\left(c_{1}^{(r)}, 0\right)$, where, in general, $c_{1}^{(r)}$ may diverge as $r \rightarrow 0+$.

Proof of Theorem 3.1. Step 1. First, we show that $\lim _{N \rightarrow+\infty} U_{u_{N}(t)}^{(N)}=S_{S(t)}$ in the $J_{1}$ topology. Writing $U_{u_{N}(t)}^{(N)}=U_{\lambda_{N} \lambda_{N}^{-1} u_{N}(t)}^{(N)}$, we note that, in light of (3.4), it is enough to show convergence in the $J_{1}$ Skorokhod topology of $S_{\lambda_{N}^{-1} u_{N}(t)}$ to $S_{S(t)}$. For any $L>0$, we exhibit increasing homeomorphisms $\Lambda_{N}:\left[0, T_{L}\right] \rightarrow\left[0, V_{L}^{(N)}\right], N \geq 1$, such that

$$
\lim _{N \rightarrow+\infty} S_{\sigma_{N}(t)}=S_{S(t)}
$$

and

$$
\lim _{N \rightarrow+\infty} \Lambda_{N}(t)=t
$$

uniformly on $\left[0, T_{L}\right]$. Here

$$
\sigma_{N}(t):=\lambda_{N}^{-1} \circ u_{N} \circ \Lambda_{N}(t)
$$

We can conclude from the above argument and from (3.4) that $\lim _{N \rightarrow+\infty}\left[U_{u_{N} \circ \Lambda_{N}(t)}^{(N)}-S_{S(t)}\right]=0$ uniformly on compact intervals.

We now display the construction of the homeomorphisms $\Lambda_{N}(t)$ that satisfy (3.11) and (3.12). Suppose that $\left\{d_{n}, n \geq 1\right\}$ is a strictly decreasing sequence, as in the statement of Lemma 3.2. Let $\left\{\ell_{k}, k \geq 1\right\}$ be an increasing sequence of positive integers such that $s_{k}:=\left\{t_{1}, \ldots, t_{\ell_{k}}\right\}=s\left(A_{d_{k}}\right)$. Then,

$$
s^{-1}\left(\delta_{k}\right)=\bigcup_{i=1}^{\ell_{k}}\left[T_{t_{i}-}, T_{t_{i}}\right] \supset A_{d_{k}} .
$$


The intervals $\left[T_{t_{i}-}, T_{t_{i}}\right]$ are the plateaus of $s(t)$. They are mutually disjoint and each is of length greater than $2 d_{k}$. The complement of $s^{-1}\left(\delta_{k}\right)$ in $\left[0, T_{L}\right]$ is an open set that is a union of a finite number of open intervals (relative to $\left[0, T_{L}\right]$ ). Let $\kappa_{k}$ be the minimum of the lengths of these intervals. Of course, $\kappa_{k}$ decreases to 0 as $k \rightarrow+\infty$. Let $\left\{m_{k}, k \geq 1\right\}$ be an increasing sequence of positive integers such that $d_{N}<\min \left[\kappa_{k} / 2, d_{k}-d_{k+1}\right]$ for all $N \geq m_{k}$. Recall that then both $\left|V_{\lambda_{N}\left(t_{i}\right)-}^{(N)}-T_{t_{i}-}\right|$ and $\left|V_{\lambda_{N}\left(t_{i}\right)}^{(N)}-T_{t_{i}}\right|, i=1, \ldots, \ell_{k}$, are less than or equal to $d_{m_{k}}$ for $N \geq m_{k}$. Therefore, for each such $N$, the intervals $\left[V_{\lambda_{N}\left(t_{i}\right)-}^{(N)}, V_{\lambda_{N}\left(t_{i}\right)}^{(N)}\right]$ (plateaus of $u_{N}$ ) are mutually disjoint for different $i=1, \ldots, \ell_{k}$.

We say that the interval $[c, d]$ follows $[a, b]$ if $c>b$. Let us take $i$ and $j$ such that their corresponding plateaus $\left[T_{t_{i}-}, T_{t_{i}}\right]$ and $\left[T_{t_{j}-}, T_{t_{j}}\right]$ are consecutive (in this order). Then $\left[V_{\lambda_{N}\left(t_{j}\right)-}^{(N)}, V_{\lambda_{N}\left(t_{j}\right)}^{(N)}\right]$ follows $\left[V_{\lambda_{N}\left(t_{i}\right)-}^{(N)}, V_{\lambda_{N}\left(t_{i}\right)}^{(N)}\right]$ for $m_{k+1}>N \geq m_{k}$. For these $N$ s we define $\Lambda_{N}\left(T_{t_{i}-}\right):=V_{\lambda_{N}\left(t_{i}\right)-}^{(N)}$ and $\Lambda_{N}\left(T_{t_{i}}\right)=V_{\lambda_{N}\left(t_{i}\right)}^{(N)}$, and elsewhere $\Lambda_{N}(t)$ is defined by a linear interpolation. It is obvious from the construction that $\Lambda_{N}(t)$ converges uniformly to $t$ on $\left[0, T_{L}\right]$ as $N \rightarrow+\infty$. Combining this with part (i) of Lemma 3.2, we also find that $\lim _{N \rightarrow+\infty} \mid \sigma_{N}(v)-$ $s(v) \mid=0$ uniformly on $\left[0, T_{L}\right]$.

Since $\Lambda_{N}(\cdot)$ dilates each $\left[T_{t_{i}-}, T_{t_{i}}\right]$ onto $\left[V_{\lambda_{N}\left(t_{i}\right)-}^{(N)}, V_{\lambda_{N}\left(t_{i}\right)}^{(N)}\right]$ with a scale greater than $d_{k+1} / d_{k}$ for any $t \in A_{d_{k}}$, we have $\Lambda_{N}(t) \in A_{d_{k+1}}$, and, thanks to Lemma 3.2, we then have, for all $N \geq m_{k+1}(\geq k+1)$,

$$
S_{\sigma_{N}(t)}=S_{S \circ \Lambda_{N}(t)}=S_{s(t)}=S_{t_{i}}
$$

see (3.13) for the definition of $\sigma_{N}(t)$. The last equality is a consequence of the fact that both $\Lambda_{N}(t)$ and $t$ belong to the same $\left[T_{t_{i}-}, T_{t_{i}}\right]$ for some $i=1, \ldots, \ell_{k}$ and $s\left(\Lambda_{N}(t)\right)=s(t)$. On the other hand, we have $u_{N} \circ \Lambda_{N}(t)=\lambda_{N}\left(t_{i}\right)$. Suppose now that $t^{\prime} \in\left[T_{t_{i}-}, T_{t_{i}}\right]$ and $t \in\left[T_{t_{i}-}, T_{t_{i}}\right] \cap A_{d_{k}}$. We then have $S_{s\left(t^{\prime}\right)}=S_{s(t)}=S_{t_{i}}$ and, since

$$
\Lambda_{N}(t), \Lambda_{N}\left(t^{\prime}\right) \in\left[V_{\lambda_{N}\left(t_{i}\right)-}^{(N)}, V_{\lambda_{N}\left(t_{i}\right)}^{(N)}\right],
$$

we have $u_{N}\left(\Lambda_{N}(t)\right)=u_{N}\left(\Lambda_{N}\left(t^{\prime}\right)\right)=\lambda_{N}\left(t_{i}\right)$. This implies that

$$
S_{\sigma_{N}\left(t^{\prime}\right)}=S_{\sigma_{N}(t)} \stackrel{(3.14)}{=} S_{S(t)}=S_{S\left(t^{\prime}\right)} .
$$

We have shown that $\lim _{N \rightarrow+\infty} S_{\sigma_{N}(t)}=S_{s(t)}$ uniformly on $s^{-1}\left(\delta_{k}\right)$ for each $k$.

The statement on the uniform convergence on the entire $\left[0, T_{L}\right]$ follows from Lemma 3.3. Indeed, suppose that $n_{k}$ is so large that

$$
\sup _{v \in\left[0, T_{L}\right]}\left(\left|\sigma_{N}(v)-s(v)\right|+\left|\Lambda_{N}(v)-v\right|\right)<d_{k} \wedge \frac{\kappa_{k}}{2} \quad \text { for } N \geq n_{k} .
$$

Also, assume that $t \notin s^{-1}\left(\delta_{k}\right)$. We claim that

$$
\text { no element from } \wp_{k} \text { lies between } \sigma_{N}(t) \text { and } s(t) \text { for } N \geq n_{k} \text {. }
$$

Indeed, suppose that $t_{i} \in s_{k}=s\left(A_{d_{k}}\right)$ and $s(t)<t_{i}<\sigma_{N}(t)$. Then, for any $v \in A_{d_{k}} \cap$ $\left[T_{t_{i}-}, T_{t_{i}}\right]$, we have $v-t>d_{k}$ and

$$
\lambda_{N}^{-1} \circ u_{N}(v)=s(v)=t_{i}<\sigma_{N}(t)=\lambda_{N}^{-1} \circ u_{N} \circ \Lambda_{N}(t),
$$

and if $v=\Lambda_{N}\left(v^{\prime}\right)$ for some $v^{\prime}$ then we have to have $v^{\prime}<t$. This however implies that $\Lambda_{N}\left(v^{\prime}\right)-v^{\prime}>v-t>d_{k}$, which is impossible in light of (3.15). The case when $s(t)>t_{i}>$ $\sigma_{N}(t)$ can be dealt with similarly. 
Suppose that $\varepsilon, \varrho>0$ are arbitrary and that $\kappa_{k}, d_{k}>0$ are sufficiently small so that, for a certain $0=s_{0}<\cdots<s_{K}=L$ that are $\kappa_{k}$-sparse, we have $\omega_{S}^{\prime}\left(\kappa_{k} ; L\right)<\varepsilon$ and

$$
\sup _{s_{i-1} \leq x, y<s_{i}}\left|S_{y}-S_{x}\right| \leq \omega_{S}^{\prime}\left(\kappa_{k} ; L\right)+\varrho .
$$

Recall here that

$$
\omega_{S}^{\prime}(\delta ; L):=\inf _{t_{i}} \sup \left[\left|S_{t}-S_{t^{\prime}}\right|, t_{i} \leq t, t^{\prime}<t_{i+1}, i=0, \ldots, N-1\right],
$$

where the infimum extends over all partitions $0=t_{0}<t_{1}<\cdots<t_{N}=L$ that are $\delta$-sparse, i.e. such that $\delta<t_{i+1}-t_{i}$ for all $i=0, \ldots, N-1$; see [6, pp. 109-110].

Then, for sufficiently large $n_{k}$, so that (3.15) and, thus, claim (3.16) also hold for any $N \geq n_{k}$ between $\sigma_{N}(t)$ and $s(t)$, there can be at most one $s_{i}$. Indeed, in the case when there were at least two such $s_{i}$ s we could estimate $\left|\sigma_{N}(t)-s(t)\right| \geq \kappa_{k}$, which would clearly contradict (3.15).

If there is no $s_{i}$ lying between $\sigma_{N}(t)$ and $s(t)$, we estimate $\left|S_{\sigma_{N}(t)}-S_{S(t)}\right| \leq \omega_{S}^{\prime}\left(\kappa_{k} ; L\right)+\varrho$. If, on the other hand, there is such a $s_{i}$ then according to (3.16) it cannot belong to $s\left(A_{d_{k}}\right)$ and we can estimate

$$
\left|S_{\sigma_{N}(t)}-S_{S(t)}\right| \leq 2\left[\omega_{S}^{\prime}\left(\kappa_{k} ; L\right)+\varrho\right]+\max _{u \notin s\left(A_{d_{k}}\right)} \Delta S_{u}<2(\varrho+\varepsilon)+\max _{u \notin s\left(A_{d_{k}}\right)} \Delta S_{u} .
$$

Summarizing, we have shown that

$$
\limsup _{N \rightarrow+\infty} \sup _{t \in\left[0, T_{L}\right]}\left|S_{\sigma_{N}(t)}-S_{s(t)}\right| \leq 2(\varrho+\varepsilon)+\max _{u \notin s\left(A_{d_{k}}\right)} \Delta S_{u}
$$

and (3.11) follows.

Step 2. Let $\sigma_{N}^{*}(t):=\lambda_{N}^{-1} \circ u_{N}^{*} \circ \Lambda_{N}(t)$, where, as we recall, $u_{N}^{*}(t)=u_{N}(t)-1 / N^{\alpha}$. Choose an arbitrary $t \in s^{-1}\left(\wp_{k}\right)$, and suppose that $t \in\left[T_{t_{i}-}, T_{t_{i}}\right] \cap A_{d_{k}}$ for some $i=1, \ldots, \ell_{k}$. Then, as we know, $\Lambda_{N}(t) \in A_{d_{k+1}} \cap\left[V_{\lambda_{N}\left(t_{i}\right)-}^{(N)}, V_{\lambda_{N}\left(t_{i}\right)}^{(N)}\right]$. Thus, $s(t)=t_{i}$ and $u_{N}^{*} \circ \Lambda_{N}(t)=$ $\lambda_{N}\left(t_{i}\right)-1 / N^{\alpha}$. We conclude therefore that

$$
S_{\sigma_{N}^{*}(t)}=S_{S(t)-c_{N}}
$$

for some $c_{N}>0$ such that $c_{N} \rightarrow 0$ as $N \rightarrow+\infty$. As a result, we obtain

$$
\lim _{N \rightarrow+\infty} S_{\sigma_{N}^{*}(t)}=\zeta_{t}^{-}
$$

for all $t \in \bigcup_{k \geq 1} s^{-1}\left(s_{k}\right)$. On the other hand, if $s(t) \notin \bigcup_{k \geq 1} s_{k}$ then, according to Lemma 3.3, we have $\Delta S_{S(t)}=0$ and, thanks to (3.11), we conclude that

$$
\lim _{N \rightarrow+\infty} S_{\sigma_{N}^{*}(t)}=\lim _{N \rightarrow+\infty} S_{\sigma_{N}(t)}=\zeta_{t}=\zeta_{t}^{-} .
$$

We have therefore shown that (3.17) holds for all $t \geq 0$ pointwise and that the limiting function is càdlàg. To complete the proof, it suffices only to observe that, thanks to (3.11), the sequence of càdlàg functions $\left\{S_{\sigma_{N}^{*}(t)}, t \geq 0\right\}$ has to converge in the $J_{1}$ topology on $D[0, L]$. Its limit has to coincide with the pointwise limit, because the set of discontinuity points of a càdlàg function is at most countable; see Corollary 12.2.1 of [29, p. 473]. This completes the proof of the theorem. 


\section{An application to a jump process arising in a quantum transport problem}

We illustrate the results obtained in the previous sections with an application to a jump process that arises in quantum mechanical transport; see [9]. Recall that the one-dimensional torus $\mathbb{T}$ is an interval $[-\pi, \pi]$ with the endpoints identified. Suppose that $\left\{K_{t}, t \geq 0\right\}$ is a jump process on $\mathbb{T}$ whose generator is given by

$$
L f(k)=\gamma(k) \int_{\mathbb{T}} \hat{r}(\theta, k)[f(\theta)-f(k)] \mathrm{d} \theta
$$

for $f \in B_{b}(\mathbb{T})$, the space of bounded Borel measurable functions on $\mathbb{T}$. The function $r_{0}^{-1} \geq \hat{r}(\theta, k) \geq r_{0}>0$ is continuous on $\mathbb{T} \times \mathbb{T}$, even and doubly stochastic, i.e. $\hat{r}(-\theta,-k)=$ $\hat{r}(\theta, k)$ and

$$
\int_{\mathbb{T}} \hat{r}(\theta, k) \mathrm{d} \theta=\int_{\mathbb{T}} \hat{r}(k, \theta) \mathrm{d} \theta=1 \quad \text { for all } k \in \mathbb{T} .
$$

On the other hand, we assume that $\gamma(k)$ is also even and strictly positive except for a possible set consisting of two points $\left\{-k_{0}, k_{0}\right\}$. More precisely, we have $\gamma(-k)=\gamma(k)$, and there is $k_{0} \in \mathbb{T}$ such that $\gamma\left(k_{0}\right)=0$ and $\inf _{\left|k-k_{0}\right| \geq \delta} \gamma(k)>0$ for any $\delta>0$. We suppose furthermore that $\gamma(k) \leq t_{*}^{-1}$ for some $t_{*}>0$ and $\int_{\mathbb{T}} \gamma^{-1}(k) \mathrm{d} k=+\infty$. This kind of process appears while considering the transport of particles in quantum systems; see, e.g. Section 4.3 of [9]. It is easy to see that $m_{*}(\mathrm{~d} k)=\gamma^{-1}(k) m_{1}(\mathrm{~d} k)$ is an infinite, reversible, invariant measure for the process. Here $m_{1}(\mathrm{~d} k)=\mathrm{d} k /(2 \pi)$ denotes the normalized Lebesgue measure on the torus. Indeed, for any $f \in B_{b}(\mathbb{T})$,

$$
\begin{aligned}
\int_{\mathbb{T}} L f(k) m_{*}(\mathrm{~d} k) & =\frac{1}{2 \pi} \int_{\mathbb{T}} \int_{\mathbb{T}} \hat{r}(\theta, k)[f(\theta)-f(k)] \mathrm{d} \theta \mathrm{d} k \\
& =\frac{1}{2 \pi} \int_{\mathbb{T}} f(\theta) \mathrm{d} \theta \int_{\mathbb{T}} \hat{r}(\theta, k) \mathrm{d} k-\frac{1}{2 \pi} \int_{\mathbb{T}} \int_{\mathbb{T}} \hat{r}(\theta, k) f(k) \mathrm{d} \theta \mathrm{d} k \\
& =0 .
\end{aligned}
$$

The process $\left\{K_{t}, t \geq 0\right\}$ can be realized using a Markov chain and a renewal process that corresponds to the jump times. Consider a skeleton Markov chain $\left\{\left(X_{n}, \rho_{n}\right), n \geq 0\right\}$, defined on $\mathbb{T}_{k_{0}} \times(0,+\infty)$, where $\mathbb{T}_{k_{0}}:=\mathbb{T} \backslash\left\{-k_{0}, k_{0}\right\},\left\{\rho_{n}, n \geq 0\right\}$ is an i.i.d. sequence of exponentially distributed random variables with intensity 1 , and $\left\{X_{n}, n \geq 0\right\}$ is an independent Markov chain with state space $\mathbb{T}_{k_{0}}$, whose transition probability equals $\hat{r}(\theta, k) \mathrm{d} \theta$. Let $\tau(k, \rho):=\gamma^{-1}(k) \rho$, $t_{0}:=0$, and $t_{n}:=\sum_{k=0}^{n-1} \tau\left(X_{k}, \rho_{k}\right), n \geq 1$. We let $K_{t}:=X_{n}$ for $t \in\left[t_{n}, t_{n+1}\right)$.

\subsection{Harris recurrence property}

Our first result concerns the recurrence property of $\left\{K_{t}, t \geq 0\right\}$.

Proposition 4.1. Suppose that $h \in\left(0, t_{*}\right)$. Consider an embedded Markov chain $\left\{K_{n h}, n \geq 0\right\}$. It is Harris recurrent with respect to the measure $m_{1}$, i.e. for any Borel subset $B$ with $m_{1}[B]>0$, we have

$$
\mathrm{P}\left[\text { there exists } n \geq 0: K_{n h} \in B\right]=1 \text {. }
$$

Proof. To simplify the notation, let $h=1$. Our hypotheses on the skeleton chain guarantee that

$$
\mathrm{P}[D]=1,
$$


where $D=\left[X_{n} \in B\right.$ infinitely often $]$. Let $A_{n}:=\left[X_{n} \in B, t_{n+1}-t_{n} \geq 2\right]$. To see that (4.2) holds, it suffices only to prove that

$$
\mathrm{P}[C]=1,
$$

where $C:=\bigcup_{n \geq 0} A_{n}$. Note that $\mathbf{1}_{C^{c}}(\omega) \leq f(\omega)$, where

$$
f(\omega):=\prod_{n \geq 0}\left[\mathbf{1}_{B^{c}}\left(X_{n}\right)+\mathbf{1}_{B}\left(X_{n}\right) \mathbf{1}_{\left\{\tau\left(X_{n}, \rho_{n}\right)<2\right\}}\right] .
$$

However,

$$
\begin{aligned}
\mathrm{E} f & =\mathrm{E}\left[\prod_{n \geq 0}\left[\mathbf{1}_{B^{c}}\left(X_{n}\right)+\mathbf{1}_{B}\left(X_{n}\right)\left(1-\mathrm{e}^{-2 \gamma\left(X_{n}\right)}\right)\right]\right] \\
& \leq \mathrm{E}\left[\prod_{n \geq 0}\left[\mathbf{1}_{B^{c}}\left(X_{n}\right)+\mathbf{1}_{B}\left(X_{n}\right)\left(1-\mathrm{e}^{-2 / t^{*}}\right)\right]\right] \\
& =\mathrm{E}\left[\prod_{n \geq 0}\left[\mathbf{1}_{B^{c}}\left(X_{n}\right)+\mathbf{1}_{B}\left(X_{n}\right)\left(1-\mathrm{e}^{-2 / t^{*}}\right)\right], D\right] \\
& =0,
\end{aligned}
$$

and (4.4) follows in light of (4.3).

As an immediate corollary of the above proposition and Theorem 1 of $[15, \mathrm{p} .116], m_{*}$ is the unique $\sigma$-finite invariant measure under the process that is absolutely continuous with respect to $m_{1}$.

Denote by $\left\{P_{t}, t \geq 0\right\}$ the transition semigroup of the process $\left\{K_{t}, t \geq 0\right\}$. It satisfies the following integral equation:

$$
P_{t} f(k)=\mathrm{e}^{-t \gamma(k)} f(k)+\gamma(k) \int_{0}^{t} \mathrm{e}^{-s \gamma(k)} \mathrm{d} s \int_{\mathbb{T}} \hat{r}\left(k^{\prime}, k\right) P_{t-s} f\left(k^{\prime}\right) \mathrm{d} k^{\prime} .
$$

For any $N \geq 1$ and $T>0$, define

$$
\Delta_{N}(T):=\left[\left(s_{0}, \ldots, s_{N-1}\right): s_{i} \geq 0, i=0, \ldots, N-1, \sum_{i=0}^{N-1} s_{i} \leq T\right] .
$$

Iterating (4.5) we can easily show that

$$
\begin{aligned}
& P_{t} f(k)= \mathrm{e}^{-t \gamma(k)} f(k) \\
&+\sum_{N=1}^{+\infty} \gamma(k) \int \ldots \iint_{\Delta_{N}(t)} \int \ldots \int_{(\mathbb{T})^{N}} \mathrm{e}^{-t \gamma\left(k_{N}\right)} \prod_{i=1}^{N}\left\{\gamma\left(k_{i}\right) \mathrm{e}^{-s_{i}\left(\gamma\left(k_{i}\right)-\gamma\left(k_{N}\right)\right)} \hat{r}\left(k_{i}, k_{i-1}\right)\right\} \\
& \times f\left(k_{N}\right) \mathrm{d} s^{(N)} \mathrm{d} k^{(N)} .
\end{aligned}
$$

Here $k_{0}:=k, \mathrm{~d} s^{(N)}:=\mathrm{d} s_{0} \cdots \mathrm{d} s_{N-1}$ and $\mathrm{d} k^{(N)}:=\mathrm{d} k_{1} \cdots \mathrm{d} k_{N}$. The component of the transition probability that is absolutely continuous with respect to $m_{1}$ therefore equals

$$
\begin{gathered}
p_{t}\left(k, k^{\prime}\right)=\sum_{N=1}^{+\infty} \gamma(k) \int \ldots \int_{\Delta_{N}(t)} \int \ldots \int_{(\mathbb{T})^{N-1}} \mathrm{e}^{-t \gamma\left(k^{\prime}\right)} \prod_{i=1}^{N}\left\{\gamma\left(k_{i}\right) \mathrm{e}^{-s_{i}\left(\gamma\left(k_{i}\right)-\gamma\left(k^{\prime}\right)\right)} \hat{r}\left(k_{i}, k_{i-1}\right)\right\} \\
\times \mathrm{d} s^{(N)} \mathrm{d} k^{(N-1)} .
\end{gathered}
$$


Here $k^{\prime}:=k_{N}$. Thus, for every $h>0, C \in \mathscr{B}\left(\mathbb{T}_{k_{0}}\right)$ with $\operatorname{dist}\left(C,\left\{-k_{0}, k_{0}\right\}\right)>0$, and $m_{1}(C)>$ 0 , we have $\inf _{k, k^{\prime} \in C} p_{h}\left(k, k^{\prime}\right)>0$. The transition probability function of any embedded chain $\left\{K_{n h}, n \geq 0\right\}$ is therefore aperiodic in the sense of [17]. Suppose that $f_{0}=\mathrm{d} v_{0} / \mathrm{d} m_{*} \in L^{2}\left(m_{*}\right)$ is a density. Thanks to the reversibility of $m_{*}$, we find that $v_{0} P_{t}$ is absolutely continuous with respect to $m_{1}$ and its density equals

$$
f_{t}=\frac{\mathrm{d} \nu_{0} P_{t}}{\mathrm{~d} m_{*}}=P_{t} f_{0} \quad \text { for all } t \geq 0 .
$$

\subsection{Mixing property of the process}

Theorem 4.1. Suppose that the initial law $v_{0}$ is absolutely continuous with respect to the Lebesgue measure $m_{1}$. Then $v_{0} P_{t}$ converges weakly as $t \rightarrow+\infty$ to the measure $\mu_{*}:=$ $1 / 2\left(\delta_{k_{0}}+\delta_{-k_{0}}\right)$. In addition, the process is completely mixing, i.e. if $v_{0}$ and $v_{0}^{\prime}$ are two absolutely continuous initial laws then

$$
\lim _{t \rightarrow+\infty}\left\|v_{0} P_{t}-v_{0}^{\prime} P_{t}\right\|_{\mathrm{TV}}=0 .
$$

To prove the above result, we first show the following.

Proposition 4.2. For any compact set $K \subset \mathbb{T}_{k_{0}}$ and a measure $v_{0}$ as in Theorem 4.1, we have

$$
\lim _{t \rightarrow+\infty} v_{0} P_{t}[K]=0 .
$$

Proof. Using a density argument, it suffices to show (4.7) for a measure $v_{0}$ whose density belongs to $L^{2}\left(m_{*}\right)$. Thanks to the strong continuity of the semigroup $\left\{P_{t}, t \geq 0\right\}$ in $L^{1}\left(m_{*}\right)$, in order to prove (4.7), it suffices to show that, for any $h>0$,

$$
\lim _{n \rightarrow+\infty} v_{0} P_{n h}[K]=0 .
$$

From the Harris recurrence property, see Proposition 4.1, we know that, for any set $A \subset \mathbb{T}_{k_{0}}$ with $m_{*}[A]>0$, we have $P_{h} \mathbf{1}_{A}(x)>0, m_{*}$-almost everywhere; hence, from [12, pp. 85-102] we have (4.8) for any $K$ such that $+\infty>m_{*}[K]>0$ (cf. Theorem C of [12, p. 91]).

Proof of Theorem 4.1. Let $h \in\left(0, t_{*}\right)$. Define $\mathcal{C}:=\bigcap_{n \geq 0} \mathcal{C}_{n}$, where $\mathcal{C}_{n}$ is the smallest $\sigma$-algebra generated by $\left\{K_{m h}, m \geq n\right\}$. According to Theorem 1 of [17, p. 45] the tail $\sigma$-algebra of the chain that is Harris recurrent and aperiodic has to be trivial. Therefore, according to Lemma 3 of [17, p. 43], (4.6) follows.

Observe that if $u(k)$ is a density with respect to $m_{*}(\mathrm{~d} k)=\gamma^{-1}(k) \mathrm{d} k$ such that $u(-k)=u(k)$ then

$$
u P_{t}(-k)=u P_{t}(k) \text {. }
$$

This follows from the fact that $v_{t}(k):=u P_{t}(-k)$ satisfies

$$
\frac{\mathrm{d} v_{t}}{\mathrm{~d} t}(k)=v_{t} L(k), \quad v_{0}(k)=u(k) .
$$

Since $u P_{t}(k)$ satisfies the same equations from the uniqueness of solutions, we obtain $u P_{t}(k)=$ $v_{t}(k)=u P_{t}(-k)$. Let $v_{0}(\mathrm{~d} k):=u(k) m_{*}(\mathrm{~d} k)$. Combining (4.9) with Proposition 4.2 and (4.6), we conclude that $v_{0} P_{t}$ converges weakly to $\frac{1}{2}\left(\delta_{-k_{0}}+\delta_{k_{0}}\right)$ as $t \rightarrow+\infty$. From the (already shown) complete mixing property we conclude in particular that, for any initial distribution $\mu_{0}$, absolutely continuous with respect to $m_{1}$, we have $\mu_{0} P_{t} \Rightarrow \frac{1}{2}\left[\delta_{-k_{0}}+\delta_{k_{0}}\right]$, weakly over $C(\mathbb{T})$, as $t \rightarrow+\infty$. 


\subsection{Limit theorems for additive functionals of the process}

In this section we will be concerned with the convergence of the laws of $N^{-\gamma} \int_{0}^{N t} \Psi\left(K_{S}\right) \mathrm{d} s$ for an appropriate $\gamma>0$ and $\Psi(k)$. Suppose that $\gamma(k) \sim c_{*}\left|k-k_{0}\right|^{\kappa}$, when $\left|k-k_{0}\right| \ll 1$, for some $\kappa>1$ and $c_{*}>0$. Then the law of $\tau(k, \rho)=\gamma^{-1}(k) \rho$ under $m_{1} \otimes \lambda$, where $\lambda(\mathrm{d} \rho):=\mathrm{e}^{-\rho} \mathrm{d} \rho$, belongs to the domain of attraction of a stable subordinator with index $\alpha=1 / \kappa$; thus, Condition 2.3 holds. One can easily verify that the other assumptions about the Markov chain made in Section 2.1 hold for $\left\{\left(X_{n}, \rho_{n}\right), n \geq 0\right\}$ as well.

Note that $\int_{0}^{t} \Psi\left(K_{s}\right) \mathrm{d} s=\hat{W}(t)$, where $\hat{W}(t)$ is the linear interpolation of a CTRW corresponding to the renewal times $\left\{t_{N}, N \geq 0\right\}$ and the partial sums $\left\{S_{N}, N \geq 0\right\}$ formed for $V(k, \rho):=\Psi(k) \tau(k, \rho)$. When $V \in L^{2}\left(m_{1} \otimes \lambda\right)$ and

$$
\int_{\mathbb{T}} \int_{0}^{+\infty} V(k, \rho) \mathrm{d} k \lambda(\mathrm{d} \rho)=0,
$$

we obtain the following corollary from Theorem 2.4 .

Corollary 4.1. The processes $\left\{Y_{t}^{(N)}:=N^{-\alpha / 2} \int_{0}^{N t} \Psi\left(K_{S}\right) \mathrm{d} s, t \geq 0\right\}$ converge over $C[0,+\infty)$ as $N \rightarrow+\infty$ to the law of the Mittag-Leffler process that corresponds to an $\alpha$-stable subordinator.

Assume also that the law of $V(k, \rho)$ under $m_{1} \otimes \lambda$ belongs to the domain of attraction of a $\beta$-stable law. Denote by $s_{N}(t)$ the right inverse of $\left\{T_{t}^{(N)}, t \geq 0\right\}$.

Corollary 4.2. Suppose that $\beta \neq 1$ and, for $\beta \in(1,2)$, that condition (4.10) holds. In addition, assume that $\tau(k, \rho)$ and $V(k, \rho)$ satisfy (2.14). Then the processes

$$
\left\{Y_{t}^{(N)}:=N^{-\alpha / \beta} \int_{0}^{N t} \Psi\left(K_{S}\right) \mathrm{d} s, t \geq 0\right\}
$$

converge in law over $\mathcal{D}$ with the $M_{1}$ topology to $\left\{\zeta_{t}:=S_{S(t)}, t \geq 0\right\}$, where $\left\{S_{t}, t \geq 0\right\}$ is a $\beta$-stable process and $s(t)$ is the right inverse of an independent, $\alpha$-stable subordinator. When $\beta=1$, the result still holds for $\left\{Y_{t}^{(N)}-c_{N} s_{N}(t), t \geq 0\right\}$, where

$$
c_{N}:=\int_{[|V(k, \rho)| \leq N]} V(k, \rho) \mathrm{d} k \lambda(\mathrm{d} \rho) .
$$

Remark. In Section 4.3 of [9] the generator of the jump process is given by $L f(k)=$ $c \cos ^{2} k \int_{\mathbb{T}} \hat{r}\left(k^{\prime}-k\right)\left[f\left(k^{\prime}\right)-f(k)\right] \mathrm{d} k^{\prime}$ for some constant $c>0$ and a density function $\hat{r}(k)$ satisfying $r_{*} \leq \hat{r}(k) \leq r_{*}^{-1}$ for some $r_{*} \in(0,1)$. Theorem 4.1 implies that $v_{0} P_{t}$ converges weakly to $1 / 2\left(\delta_{-\pi / 2}+\delta_{\pi / 2}\right)$ as $t \rightarrow+\infty$ for any initial measure $v_{0}$ absolutely continuous with respect to the Lebesgue measure. This answers in the affirmative the conjecture made in [9]. For an observable $\Psi$ such that $V=\Psi \tau \in L^{2}\left(m_{1} \otimes \lambda\right)$, the functionals $\left\{N^{-1 / 4} \int_{0}^{N t} V\left(K_{s}\right) \mathrm{d} s, t \geq 0\right\}$ converge in law to a Mittag-Leffler process. Note that when $\Psi(-k)=-\Psi(k)$ belongs to the normal domain of attraction of a Cauchy law and it is not singular at $\pi / 2$, then the processes $\left\{N^{-1 / 2} \int_{0}^{N t} \Psi\left(K_{s}\right) \mathrm{d} s, t \geq 0\right\}$ converge in law as $N \rightarrow+\infty$ to a process that has the same scaling properties as a Brownian motion but is not Markovian (recall that we call it a fake diffusion). 


\section{Appendix A. The proofs of Theorems 2.1 and 2.2}

Theorems 2.1 and 2.2 can be proved from the result we formulate below. It is essentially a two-dimensional version of Theorem 4.1 of [11, p. 840]. Since its proof is a simple modification of an argument presented ibid., we omit its presentation here. Also, for simplicity, we only consider the case when $K_{N}=N$.

Before formulating the result we introduce some notation. Suppose that $\left\{Z_{n, N}, n \geq 0\right.$, $N \geq 1\}$ is an array of $\mathbb{R}^{2}$-valued random vectors on $(\Omega, \mathcal{F}, \mathrm{P})$ and that $\left\{g_{n, N}, n \geq-1, N \geq 1\right\}$ is an array of sub- $\sigma$-algebras of $\mathcal{F}$ such that $g_{n-1, N} \subset g_{n, N}, N \geq 1, n \geq 0$. For a fixed $\Delta=\left(\Delta_{1}, \Delta_{2}\right) \in(0,+\infty)^{2}$, define

$$
Z_{t}^{(\Delta, N)}:=\sum_{n=0}^{[N t]-1} Z_{n, N}^{(\Delta)}
$$

where

$$
Z_{n, N}^{(\Delta)}:=Z_{n, N} \mathbf{1}_{\left\{\left|Z_{n, N}^{(i)}\right| \leq \Delta_{i}, i=1,2\right\}}-\mathrm{E}\left[Z_{n, N} \mathbf{1}_{\left\{\left|Z_{n, N}^{(i)}\right| \leq \Delta_{i}, i=1,2\right\}} \mid g_{n-1, N}\right]
$$

and

$$
\tilde{Z}_{t}^{(\Delta, N)}:=\sum_{n=0}^{[N t]-1}\left[Z_{n, N}-\mathrm{E}\left[Z_{n, N} \mathbf{1}_{\left\{\left|Z_{n, N}^{(i)}\right| \leq \Delta_{i}, i=1,2\right\}} \mid g_{n-1, N}\right]\right] .
$$

Let $\left|\left(x_{1}, x_{2}\right)\right|_{\infty}:=\max \left\{\left|x_{1}\right|,\left|x_{2}\right|\right\}$. Suppose that $\nu_{0}$ is a measure on $\mathbb{R}_{*}^{2}:=\mathbb{R}^{2} \backslash\{(0,0)\}$ such that $\int_{\mathbb{R}_{*}^{2}}\left(|x|^{2} \wedge 1\right) v_{0}(\mathrm{~d} x)<+\infty$. To simplify the statement, we assume that $v_{0}$ is absolutely continuous with respect to the two-dimensional Lebesgue measure.

Theorem A.1. (See [11].) Assume that, for any $g \in C_{b}^{\infty}\left(\mathbb{R}_{*}^{2}\right)$ such that $0 \notin \operatorname{supp} g$, we have

$$
\begin{gathered}
\lim _{N \rightarrow+\infty} \mathrm{E}\left|\sum_{n=0}^{N} \mathrm{E}\left[g\left(Z_{n, N}\right) \mid g_{n-1, N}\right]-\int_{\mathbb{R}_{*}^{2}} g\left(\lambda_{1}, \lambda_{2}\right) v_{0}\left(\mathrm{~d} \lambda_{1}, \mathrm{~d} \lambda_{2}\right)\right|=0, \\
\lim _{N \rightarrow+\infty} \sum_{n=0}^{N} \mathrm{E}\left(\mathrm{E}\left[g\left(Z_{n, N}\right) \mid g_{n-1, N}\right]\right)^{2}=0,
\end{gathered}
$$

and

$$
\lim _{|\Delta|_{\infty} \rightarrow 0+} \limsup _{N \rightarrow+\infty} E\left[\sup _{t \in[0, T]}\left|Z_{t}^{(\Delta, N)}\right|_{\infty}^{2}\right]=0
$$

for any $T>0$. Then, for each $\Delta=\left(\Delta_{1}, \Delta_{2}\right) \in(0,+\infty)^{2}$, the processes $\left\{\tilde{Z}_{t}^{(\Delta, N)}, t \geq 0\right\}$ converge in law over $\mathscr{D}_{2}$, with the $J_{1}$-topology to the Lévy process $\left\{Z_{t}=\left(Z_{t}^{(1)}, Z_{t}^{(2)}\right), t \geq 0\right\}$ such that $\mathrm{Ee}^{i \xi_{1} Z_{t}^{(1)}+i \xi_{2} Z_{t}^{(2)}}=\mathrm{e}^{t \psi\left(\xi_{1}, \xi_{2}\right)}$ and

$$
\begin{aligned}
\psi\left(\xi_{1}, \xi_{2}\right):= & \int_{\bigcup_{i=1}^{2}\left[\left|\lambda_{i}\right| \geq \Delta_{i}\right]}\left(\mathrm{e}^{i \xi_{1} \lambda_{1}+i \xi_{2} \lambda_{2}}-1\right) \nu_{0}\left(\mathrm{~d} \lambda_{1}, \mathrm{~d} \lambda_{2}\right) \\
& +\int_{\left[\left|\lambda_{i}\right| \leq \Delta_{i}, i=1,2\right]}\left[\mathrm{e}^{i \xi_{1} \lambda_{1}+i \xi_{2} \lambda_{2}}-1-i\left(\lambda_{1} \xi_{1}+\lambda_{2} \xi_{2}\right)\right] \nu_{0}\left(\mathrm{~d} \lambda_{1}, \mathrm{~d} \lambda_{2}\right) .
\end{aligned}
$$

To use the above theorem, we transform slightly the process $\left\{X_{t}^{(N)}:=\left(S_{t}^{(N)}, T_{t}^{(N)}\right), t \geq 0\right\}$. In order to simplify the notation, we consider only the $\beta \in(1,2)$ case; the $\beta \in(0,1]$ case can 
be proved similarly (in fact, it is even simpler then). Suppose that $\gamma \in(1, \beta)$. Thanks to the spectral gap condition (2.1), we can find a unique zero-mean solution $\chi$ in $L^{\gamma}(\pi)$ of

$$
(I-P) \chi=V .
$$

For a fixed $M>0$, we let $M_{N}:=M N^{1 / \alpha}$ and

$$
\tau^{(N)}(x):=\tau(x) \mathbf{1}_{\left\{\tau(x)<M_{N}\right\}} .
$$

We also let $Z_{n, N}(M):=\left(Z_{n, N}^{(1)}, Z_{n, N}^{(2)}(M)\right)$, where

$$
Z_{0, N}^{(1)}:=0, \quad Z_{n, N}^{(1)}:=\frac{1}{N^{1 / \beta}} R_{0}\left(X_{n}, X_{n-1}\right), \quad n \geq 1 .
$$

Here $R_{0}(x, y):=\chi(x)-P \chi(y)$. In addition, $Z_{n, N}^{(2)}(M):=N^{-1 / \alpha} \tau^{(N)}\left(X_{n}\right), n \geq 0$. For $N, n \geq 0$, we let $g_{n, N}$ be the $\sigma$-algebra generated by $X_{0}, \ldots, X_{n}$. By convention we let $g_{-1, N}$ be the trivial $\sigma$-algebra.

Define the process

$$
Z_{t}^{(N)}(M)=\left(Z_{t, N}^{(1)}, Z_{t, N}^{(2)}(M)\right):=\sum_{n=0}^{[N t]-1} Z_{n, N}(M), \quad t \geq 0 .
$$

A simple calculation shows that

$$
X_{t}^{(N)}-Z_{t}^{(N)}(M)=\left(N^{-1 / \beta}\left[\chi\left(X_{0}\right)-P \chi\left(X_{[N t]-1}\right)\right], N^{-1 / \alpha} \sum_{n=0}^{[N t]-1} \tau\left(X_{n}\right) \mathbf{1}_{\left\{\tau\left(X_{n}\right)>M_{N}\right\}}\right) .
$$

Hence, due to (A.12) below and (2.5), for any $T, \sigma>0$, we have

$$
\lim _{M \rightarrow+\infty} \limsup _{N \rightarrow+\infty} \mathrm{P}\left[\sup _{t \in[0, T]}\left|X_{t}^{(N)}-Z_{t}^{(N)}(M)\right|_{\infty} \geq \sigma\right]=0 .
$$

To prove Theorems 2.1 and 2.2, it suffices therefore to show the convergence of the processes $\left\{Z_{t}^{(N)}(M), t \geq 0\right\}, N \geq 1$, for a fixed $M>0$ to a Lévy process $\left\{Z_{t}(M), t \geq 0\right\}$ whose exponent equals

$$
\psi\left(\xi_{1}, \xi_{2}\right)=\int_{\mathbb{R}^{2}}\left[\mathrm{e}^{i \xi_{1} \lambda_{1}+i \xi_{2} \lambda_{2}}-1-i\left(\lambda_{1} \xi_{1}+\lambda_{2} \xi_{2}\right)\right] \mathbf{1}_{\left\{\left|\lambda_{2}\right| \leq M\right\}} \nu_{*}\left(\mathrm{~d} \lambda_{1}, \mathrm{~d} \lambda_{2}\right)
$$

From now on we drop $M$ from our subsequent notation of stochastic processes, writing $Z_{n, N}:=Z_{n, N}(M), Z_{t}^{(N)}:=Z_{t}^{(N)}(M)$, and $Z_{t}:=Z_{t}(M)$.

Below we verify that the processes $\left\{Z_{t}^{(N)}, t \geq 0\right\}, N \geq 1$, satisfy the assumptions of Theorem A.1 with the measure $v_{0}$ equal to $v_{*}$ given by (2.13), or (2.18) correspondingly. Accepting this claim for a moment we show how to reach the conclusions of the aforementioned theorems. Let $\Delta_{2}:=M$. From Theorem A.1 we deduce that the processes

$$
\tilde{Z}_{t}^{(N)}\left(\Delta_{1}\right):=Z_{t}^{(N)}-\sum_{n=0}^{[N t]-1} \mathrm{E}\left[Z_{n, N} \mathbf{1}_{\left\{\left|Z_{n, N}^{(1)}\right| \leq \Delta_{1}\right\}} \mathbf{1}_{\left\{\left|Z_{n, N}^{(2)}\right| \leq \Delta_{2}\right\}} \mid g_{n-1, N}\right], \quad t \geq 0,
$$


converge in law over $\mathcal{D}_{2}$ to a Lévy process $\left\{Z_{t}, t \geq 0\right\}$ whose exponent is equal to $\psi\left(\xi_{1}, \xi_{2}\right)$ given by (A.4) (with $\Delta_{2}=M$ ). Since $\mathrm{E}\left[Z_{n, N}^{(1)} \mid g_{n-1, N}\right]=0$ and $\left|Z_{n, N}^{(2)}\right| \leq M$ a.s., we obtain

$$
\sum_{n=0}^{[N t]-1} \mathrm{E}\left[Z_{n, N}^{(1)} \mathbf{1}_{\left\{\left|Z_{n, N}^{(i)}\right| \leq \Delta_{i}, i=1,2\right\}} \mid g_{n-1, N}\right]=-\sum_{n=0}^{[N t]-1} \mathrm{E}\left[Z_{n, N}^{(1)} \mathbf{1}_{\left\{\left|Z_{n, N}^{(1)}\right| \geq \Delta_{1}\right\}} \mid g_{n-1, N}\right] .
$$

This, together with (A.11) and (A.12) below, and hypothesis (2.5), implies that, for any $T>0$, we have

$$
\lim _{\Delta_{1} \rightarrow+\infty} \limsup _{N \rightarrow+\infty} \mathrm{E}\left|\sup _{t \in[0, T]} \sum_{n=0}^{[N t]-1} \mathrm{E}\left[Z_{n, N} \mathbf{1}_{\left\{\left|Z_{n, N}^{(1)}\right| \leq \Delta_{1}\right\}} \mathbf{1}_{\left\{\left|Z_{n, N}^{(2)}\right| \leq M\right\}} \mid g_{n-1, N}\right]\right|=0 .
$$

Letting $\Delta_{1} \rightarrow+\infty$, we deduce that the processes $\left\{Z_{t}^{(N)}, t \geq 0\right\}$ converge in law over $\mathscr{D}_{2}$ to a Lévy process with Lévy exponent (A.9).

We start with the following lemma which, among other things, allows us to justify the limits in (A.8) and (A.10).

Lemma A.1. We have

$$
\pi(\chi \geq \lambda)=\frac{c_{\beta}^{+}}{\lambda^{\beta}}(1+o(1)), \quad \pi(\chi \leq-\lambda)=\frac{c_{\beta}^{-}}{\lambda^{\beta}}(1+o(1)), \quad \text { as } \lambda \rightarrow+\infty .
$$

In addition,

$$
\|P|\chi|\|_{L^{2}(\pi)}<+\infty
$$

and there exists $C>0$ such that

$$
\left\|P \tau^{(N)}\right\|_{L^{2}(\pi)} \leq C N^{1 / \alpha-1} \text { for all } N \geq 1, M>0 .
$$

Proof. Observe first that

$$
\begin{aligned}
\|P|V|\|_{L^{2}(\pi)}^{2} & \stackrel{(2.10)}{\leq} C \int\left(\int_{0}^{+\infty} P_{a}(x,|V|>\lambda) \mathrm{d} \lambda\right)^{2} \pi(\mathrm{d} x) \\
& =C \int\left(\int p(x, y)|V(y)| \pi(\mathrm{d} y)\right)^{2} \pi(\mathrm{d} x) \\
& \stackrel{\text { Jensen }}{\leq} C \int\left(\int p^{2}(x, y) \frac{|V(y)| \pi(\mathrm{d} y)}{\|V\|_{L^{1}(\pi)}}\right) \pi(\mathrm{d} x)\|V\|_{L^{1}(\pi)}^{2} \\
& \leq C C(2)\|V\|_{L^{1}(\pi)}^{2} .
\end{aligned}
$$

Since $\chi=(I-P)^{-1} V=\sum_{n \geq 0} P^{n} V$ from the above, we deduce that $P|\chi| \leq(I-P)^{-1} P|V|$; thus $P|\chi| \in L^{2}(\pi)$. From this and the Poisson equation (A.5), it follows that $\chi$ satisfies (A.11).

To show (A.13), we write

$$
\begin{aligned}
\left\|P \tau^{(N)}\right\|_{L^{2}(\pi)}^{2} & \leq C(2)\left\|\tau^{(N)}\right\|_{L^{1}(\pi)}^{2} \\
& \leq C(2)\left(\int_{0}^{M_{N}} \pi[\tau>\lambda] \mathrm{d} \lambda\right)^{2} \\
& \leq C\left(\int_{0}^{M_{N}} \frac{\mathrm{d} \lambda}{1+\lambda^{\alpha}}\right)^{2} \\
& \leq C^{\prime} N^{2(1 / \alpha-1)}
\end{aligned}
$$




\section{A.1. Proof of (A.1)}

Since

$$
\begin{aligned}
& \lim _{\Delta_{1} \rightarrow+\infty} \limsup _{N \rightarrow+\infty} \mathrm{E} \mid \sup _{t \in[0, T]} \sum_{n=0}^{[N t]-1} \mathrm{P}\left[\left|Z_{n, N}^{(1)}\right| \geq \Delta_{1} \text { or }\left|Z_{n, N}^{(2)}\right| \geq M \mid g_{n-1, N}\right] \mid \\
& \quad=\lim _{\Delta_{1} \rightarrow+\infty} \limsup _{N \rightarrow+\infty} \mathrm{E}\left|\sup _{t \in[0, T]} \sum_{n=0}^{[N t]-1} \mathrm{P}\left[\left|Z_{n, N}^{(1)}\right| \geq \Delta_{1} \mid g_{n-1, N}\right]\right| \\
& \quad=0
\end{aligned}
$$

it suffices to show (A.1) for $g \in C_{0}^{\infty}\left(\mathbb{R}_{*}^{2}\right)$. In that case we can expand $g\left(Z_{n+1, N}\right)$ using Taylor's formula around $z^{(N)}\left(X_{n+1}\right)$, where $z^{(N)}(x):=\left(N^{-1 / \beta} V(x), N^{-1 / \alpha} \tau^{(N)}(x)\right)$, and obtain

$$
\begin{aligned}
\sum_{n=0}^{N-1} \mathrm{E}\left[g\left(Z_{n+1, N}\right) \mid g_{n}\right]= & \sum_{n=0}^{N-1} \mathrm{E}\left[g\left(z^{(N)}\left(X_{n+1}\right)\right) \mid g_{n}\right] \\
& +\sum_{n=0}^{N-1} \int_{0}^{1} \mathrm{E}\left[R\left(X_{n+1}, X_{n}\right) \nabla g\left(z_{n}^{(N)}(\lambda)\right) \mid g_{n}\right] \mathrm{d} \lambda .
\end{aligned}
$$

Here $z_{n}^{(N)}(\lambda):=\lambda R\left(X_{n+1}, X_{n}\right)+z^{(N)}\left(X_{n+1}\right)$ and

$$
R(x, y):=\left(N^{-1 / \beta}[P \chi(x)-P \chi(y)],-N^{-1 / \alpha} P \tau^{(N)}(y)\right) .
$$

Denote the first and second terms on the right-hand side of (A.14) by $I_{N}$ and $I I_{N}$, respectively. We can write $I_{N}=I_{N}^{(1)}+I_{N}^{(2)}$, where

$$
\begin{aligned}
I_{N}^{(1)} & :=\sum_{n=1}^{N} \int g\left(z^{(N)}(y)\right) P\left(X_{n-1}, \mathrm{~d} y\right)-N \int g\left(z^{(N)}(x)\right) \pi(\mathrm{d} x), \\
I_{N}^{(2)} & :=N \int g\left(z^{(N)}(x)\right) \pi(\mathrm{d} x) .
\end{aligned}
$$

Note that

$$
\mathrm{E}\left|I_{N}^{(1)}\right|=\mathrm{E}\left|\sum_{n=1}^{N} P G_{N}\left(X_{n-1}\right)\right|,
$$

where $G_{N}(x):=g\left(z^{(N)}(x)\right)-\int g\left(z^{(N)}(y)\right) \pi(\mathrm{d} y)$. Let $u_{N}:=(I-P)^{-1} P G_{N}$. By the spectral gap condition (2.2) we have

$$
\int u_{N}^{2}(x) \pi(\mathrm{d} x) \leq \frac{1}{1-a^{2}} \int\left(P G_{N}\right)^{2}(x) \pi(\mathrm{d} x) .
$$

Let $a_{*}:=\operatorname{dist}(0, \operatorname{supp} g)$. Note that

$$
\begin{aligned}
\int\left(P G_{N}\right)^{2}(x) \pi(\mathrm{d} x) \leq 2\|g\|_{\infty}^{2}[ & \int\left(\int_{\left[\tau(y) \geq a^{*} N^{1 / \alpha} / 2\right]} p(x, y) \pi(\mathrm{d} y)\right)^{2} \pi(\mathrm{d} x) \\
& \left.+\int\left(\int_{\left[|V(y)| \geq a^{*} N^{1 / \beta} / 2\right]} p(x, y) \pi(\mathrm{d} y)\right)^{2} \pi(\mathrm{d} x)\right]
\end{aligned}
$$




$$
\begin{aligned}
& \leq 2 C\|g\|_{\infty}^{2} N^{-1}\left[\iint_{\left[\tau(y) \geq a^{*} N^{1 / \alpha} / 2\right]} p^{2}(x, y) \pi(\mathrm{d} y) \pi(\mathrm{d} x)\right. \\
& \left.\quad+\iint_{\left[|V(y)| \geq a^{*} N^{1 / \beta} / 2\right]} p^{2}(x, y) \pi(\mathrm{d} y) \pi(\mathrm{d} x)\right] \\
& \leq 2 C\|g\|_{\infty}^{2} N^{-1} o(1)
\end{aligned}
$$

as $N \rightarrow+\infty$. In addition, we can rewrite

$$
\sum_{n=1}^{N} P G_{N}\left(X_{n-1}\right)=u_{N}\left(X_{0}\right)-u_{N}\left(X_{[N t]}\right)+\sum_{n=1}^{[N t]-1} U_{n},
$$

where $U_{n}:=u_{N}\left(X_{n}\right)-P u_{N}\left(X_{n-1}\right), n \geq 1$, is a stationary sequence of martingale differences with respect to $\left\{g_{n}, n \geq 0\right\}$. Consequently,

$$
\mathrm{E}\left|I_{N}^{(1)}\right| \leq\left(\mathrm{E}\left[\sum_{n=1}^{N} P G_{N}\left(X_{n-1}\right)\right]^{2}\right)^{1 / 2} \leq C\left(N \int\left(P G_{N}\right)^{2}(y) \pi(\mathrm{d} y)\right)^{1 / 2} \rightarrow 0,
$$

by virtue of (A.15). To prove that

$$
\lim _{N \rightarrow+\infty} \mathrm{E}\left|I_{N}-\int g\left(\lambda_{1}, \lambda_{2}\right) \mathbf{1}_{[0, M]}\left(\lambda_{2}\right) \nu_{*}\left(\mathrm{~d} \lambda_{1}, \mathrm{~d} \lambda_{2}\right)\right|=0,
$$

it suffices to use the argument above and the following result.

Proposition A.1. Suppose that $g \in C_{0}^{\infty}\left(\mathbb{R}_{*}^{2}\right)$. Then, under the assumptions of either Theorem 2.1 or 2.2 , we have

$$
\lim _{N \rightarrow+\infty} I_{N}^{(2)}=\int g\left(\lambda_{1}, \lambda_{2}\right) \mathbf{1}_{[0, M]}\left(\lambda_{2}\right) v_{*}\left(\mathrm{~d} \lambda_{1}, \mathrm{~d} \lambda_{2}\right),
$$

where $\nu_{*}\left(\mathrm{~d} \lambda_{1}, \mathrm{~d} \lambda_{2}\right)$ is given by (2.13) or (2.18), respectively.

Proof. Case 1: (2.14) holds. Suppose that $\gamma>\kappa_{1}>\alpha \vee \beta$, where $\gamma$ is the same as in (2.14), and that

$$
\begin{aligned}
& A_{N}:=\left[\tau^{(N)} \geq \frac{a_{*}}{2} N^{1 / \kappa_{1}},|V| \geq \frac{a_{*}}{2} N^{1 / \beta}\right], \\
& B_{N}:=\left[\tau^{(N)} \geq \frac{a_{*}}{2} N^{1 / \alpha},|V| \geq \frac{a_{*}}{2} N^{1 / \kappa_{1}}\right] .
\end{aligned}
$$

Observe that $\pi\left(A_{N}\right)=o(1 / N)$ and $\pi\left(B_{N}\right)=o(1 / N)$. To compute the limit in (A.16), it suffices therefore to compute $\lim _{N \rightarrow+\infty} \mathcal{K}_{N}^{(i)}, i=1,2$, where

$$
\mathcal{K}_{N}^{(i)}:=N \int g\left(z^{(N)}(x)\right) \mathbf{1}_{C_{N}^{(i)}} \pi(\mathrm{d} x), \quad i=1,2,
$$

and

$$
\begin{aligned}
C_{N}^{(1)} & :=\left[\tau^{(N)} \leq \frac{a_{*}}{2} N^{1 / \kappa_{1}},|V| \geq \frac{a_{*}}{2} N^{1 / \beta}\right], \\
C_{N}^{(2)} & :=\left[\tau^{(N)} \geq \frac{a_{*}}{2} N^{1 / \alpha},|V| \leq \frac{a_{*}}{2} N^{1 / \kappa_{1}}\right] .
\end{aligned}
$$


Up to a term of order $o(1)$, we have $\mathcal{K}_{N}^{(i)}=\tilde{\mathcal{K}}_{N}^{(i)}$, where $\tilde{\mathcal{K}}_{N}^{(i)}:=N \int g\left(\tilde{z}_{i}^{(N)}(x)\right) \pi(\mathrm{d} x)$, and $\tilde{z}_{1}^{(N)}(x):=\left(N^{-1 / \beta} V(x), 0\right), \tilde{z}_{2}^{(N)}(x):=\left(0, N^{-1 / \alpha} \tau^{(N)}(x)\right)$. We can write

$$
\begin{aligned}
\lim _{N \rightarrow+\infty} \tilde{\mathcal{K}}_{N}^{(1)}= & \lim _{N \rightarrow+\infty} N^{1-1 / \beta} \int_{0}^{+\infty} \partial_{1} g\left(N^{-1 / \beta} \lambda, 0\right) \pi(V>\lambda) \mathrm{d} \lambda \\
& +\lim _{N \rightarrow+\infty} N^{1-1 / \beta} \int_{0}^{+\infty} \partial_{1} g\left(-N^{-1 / \beta} \lambda, 0\right) \pi(V<-\lambda) \mathrm{d} \lambda \\
& \stackrel{(2.9)}{=} \int_{-\infty}^{+\infty} \partial_{1} g(\lambda, 0) \frac{c_{\beta}(\lambda)}{|\lambda|^{\beta}} \mathrm{d} \lambda .
\end{aligned}
$$

Likewise,

$$
\lim _{N \rightarrow+\infty} \tilde{\mathcal{K}}_{N}^{(2)}=\int_{0}^{M} \partial_{2} g(0, \lambda) \frac{c_{\alpha}(\lambda)}{\lambda^{\alpha}} \mathrm{d} \lambda,
$$

and (A.16) follows.

Case 2: (2.16) holds. We will need the following lemma.

Lemma A.2. Suppose that (2.16) holds. Then there exists a constant $C_{*}$ such that

$$
\pi[|\tau-\rho \circ \chi| \geq \lambda] \leq \frac{C_{*}}{\lambda \gamma} .
$$

The exponent $\gamma$ is the same as in (2.16).

Proof. The left-hand side of (A.17) can be estimated by

$$
\pi\left[|\tau-\rho \circ V| \geq \frac{1}{2} \lambda\right]+\pi\left[|\rho \circ V-\rho \circ \chi| \geq \frac{1}{2} \lambda\right] .
$$

The first term can be estimated directly from (2.16). When $\beta \leq \alpha$, we have $\left|\rho\left(\lambda_{1}\right)-\rho\left(\lambda_{2}\right)\right| \leq$ $\rho\left(\lambda_{1}-\lambda_{2}\right)$ for all $\lambda_{1}, \lambda_{2} \in \mathbb{R}$. The second term in (A.18) can be estimated from (A.12) by

$$
\pi\left[|P \chi| \geq\left(\frac{\lambda}{2}\right)^{\alpha / \beta}\right] \leq \frac{C\|P \chi\|_{L^{2}(\pi)}^{2}}{\lambda^{2 \alpha / \beta}}
$$

and (A.17) follows for $\beta \in(0,2)$.

When, on the other hand, $\beta>\alpha$, the second term in (A.18) can be estimated by

$$
\begin{aligned}
& \pi\left[\left|\max \left\{e^{\prime} \circ V, e^{\prime} \circ \chi\right\}\right||(V-\chi)| \geq \lambda\right] \\
& \quad \leq \pi\left[\left|e^{\prime} \circ V P \chi\right| \geq \frac{1}{2} \lambda\right]+\pi\left[\left|e^{\prime} \circ \chi P \chi\right| \geq \frac{1}{2} \lambda\right] .
\end{aligned}
$$

To estimate the first term on the right-hand side, we recall that, according to Young's inequality, $\lambda_{1} \lambda_{2} \leq \lambda_{1}^{p} / p+\lambda_{2}^{q} / q$ for any $\lambda_{1}, \lambda_{2}>0$ and $p, q>0$ such that $p^{-1}+q^{-1}=1$. Choose $p$ such that $p_{1}:=p(\beta / \alpha-1)<\beta / \alpha$ and $(\beta+2) /(2 \alpha)>q>\beta / \alpha$. The first term in (A.19) can be estimated by

$$
\pi\left[|V| \geq C_{1} \lambda^{1 / p_{1}}\right]+\pi\left[|P \chi| \geq C_{2} \lambda^{1 / q}\right]
$$

for some constants $C_{1}, C_{2}>0$ independent of $\lambda$. The first term can be estimated by $C \lambda^{-\beta / p_{1}}$, while the second can be estimated by $C \lambda^{-2 / q}\|P \chi\|_{L^{2}(\pi)}$. These together yield the desired bound on the first term in (A.19). The second term can be dealt with similarly. 
Let

$$
A_{N}:=\left[|V| \geq \frac{1}{2} a_{*} N^{1 / \beta} / 2 \text { or } \tau^{(N)} \geq \frac{1}{2} a_{*} N^{1 / \alpha}\right],
$$

and, for some $\gamma>\kappa>\alpha$, let $B_{N}:=\left[|\rho \circ V-\tau| \geq N^{1 / \kappa}\right]$. Observe that, from (2.14), (2.16), and Lemma A.2,

$$
\pi\left(A_{N}\right) \leq \frac{C}{N} \quad \text { and } \quad \pi\left(B_{N}\right) \leq \frac{C}{N^{\gamma / \kappa}} .
$$

Also, let $\rho^{(N)}(x):=\rho(x) \mathbf{1}_{\left\{\rho<M_{N}\right\}}(x)$. Define

$$
\tilde{z}^{(N)}(x):=\left(N^{-1 / \beta} V(x), N^{-1 / \alpha} \rho^{(N)} \circ V(x)\right) .
$$

Note that $z^{(N)}(x)=\tilde{z}^{(N)}(x)+r^{(N)}(x)$, where

$$
r^{(N)}(x):=\left(0, N^{-1 / \alpha}\left[\tau^{(N)}(x)-\rho^{(N)} \circ V(x)\right]\right) .
$$

Note that $z^{(N)}(x)$ lies outside the support of $g$ on $A_{N}^{c}$. Therefore, the expression under the limit in (A.16) can be written as

$$
N \int g\left(z^{(N)}(x)\right) \mathbf{1}_{A_{N}} \pi(\mathrm{d} x)=\mathfrak{I}_{N}+g_{N},
$$

where

$$
\tau_{N}:=N \int g\left(z^{(N)}(x)\right) \mathbf{1}_{A_{N}} \mathbf{1}_{B_{N}} \pi(\mathrm{d} x), \quad g_{N}:=N \int g\left(z^{(N)}(x)\right) \mathbf{1}_{A_{N}} \mathbf{1}_{B_{N}^{c}} \pi(\mathrm{d} x) .
$$

Note that

$$
I_{N} \leq N\|g\|_{\infty} \pi\left(B_{N}\right) \stackrel{(\mathrm{A} .20)}{\leq} C N^{1-\gamma / \kappa}\|g\|_{\infty} \rightarrow 0 \quad \text { as } N \rightarrow+\infty .
$$

Finally, $g_{N}=g_{N}^{(1)}+g_{N}^{(2)}$, where

$$
\begin{aligned}
\mathcal{g}_{N}^{(1)} & :=N \int g\left(\tilde{z}^{(N)}(x)\right) \mathbf{1}_{A_{N}} \mathbf{1}_{B_{N}^{c}} \pi(\mathrm{d} x), \\
g_{N}^{(2)} & :=N \iint_{0}^{1} \nabla g\left(\tilde{z}^{(N)}(x)+\lambda r^{(N)}(x)\right) r^{(N)}(x) \mathbf{1}_{A_{N}} \mathbf{1}_{B_{N}^{c}} \pi(\mathrm{d} x) \mathrm{d} \lambda .
\end{aligned}
$$

Given $\delta>0$, we choose $N_{0}$ so that, for $N \geq N_{0}$, we have $N^{1 / \kappa}<\delta N^{1 / \alpha}$. Let

$$
\begin{aligned}
& C_{N}^{(1)}:=\left[\rho \circ V<M N^{1 / \alpha},(M+\delta) N^{1 / \alpha} \leq \tau\right], \\
& C_{N}^{(2)}:=\left[\tau<M N^{1 / \alpha},(M+\delta) N^{1 / \alpha} \leq \rho \circ V\right],
\end{aligned}
$$

and $D_{N}:=\left[N^{-1 / \alpha} \tau \in(M-\delta, M+\delta)\right]$. Note that (recall that $M_{N}:=M N^{1 / \alpha}$ )

$$
\begin{aligned}
& B_{N}^{c} \cap\left(C_{N}^{(1)} \cup C_{N}^{(2)} \cup D_{N}\right)^{c} \subset E_{N} \\
& \quad:=\left(B_{N}^{c} \cap\left[\rho \circ V \leq M_{N}, \tau \leq M_{N}\right]\right) \cup\left(B_{N}^{c} \cap\left[\rho \circ V \geq M_{N}, \tau \geq M_{N}\right]\right) .
\end{aligned}
$$

We have

$$
\begin{aligned}
\mathscr{g}_{N}^{(2)} & \leq N\|\nabla g\|_{\infty} \int\left|r^{(N)}(x)\right| \mathbf{1}_{A_{N}} \mathbf{1}_{B_{N}^{c}} \pi(\mathrm{d} x) \\
& \leq C N^{1+1 / \kappa-1 / \alpha}\|\nabla g\|_{\infty} \pi\left(A_{N}\right)+N\|\nabla g\|_{\infty}\left[\pi\left(C_{N}^{(1)}\right)+\pi\left(C_{N}^{(2)}\right)+\pi\left(D_{N}\right)\right] .
\end{aligned}
$$


The first term on the right-hand side comes from the estimate $\left|r^{(N)}(x)\right| \leq N^{1 / \kappa-1 / \alpha}$ that holds on $E_{N}$. The remaining terms can be estimated by

$$
c_{\alpha}(1+o(1))\|\nabla g\|_{\infty}\left[(M-\delta)^{-\alpha}-(M+\delta)^{-\alpha}\right],
$$

where $o(1) \rightarrow 0$ as $N \rightarrow+\infty$. This expression can be made arbitrarily small when $\delta>0$ is chosen sufficiently small.

Concerning the term $g_{N}^{(1)}$, we can repeat the above argument and justify in that way that it is equal, up to a term of order $o(1)$, to

$$
\begin{aligned}
\tilde{\mathscr{g}}_{N}^{(1)}:= & N \int g\left(\tilde{z}^{(N)}(x)\right) \pi(\mathrm{d} x) \\
= & N \iint_{0}^{+\infty} \frac{\mathrm{d}}{\mathrm{d} \lambda} g\left(N^{-1 / \beta} \lambda, N^{-1 / \alpha} \rho^{(N)}(\lambda)\right) \mathbf{1}_{\{0<\lambda<V(x)\}} \pi(\mathrm{d} x) \mathrm{d} \lambda \\
& -N \iint_{-\infty}^{0} \frac{\mathrm{d}}{\mathrm{d} \lambda} g\left(N^{-1 / \beta} \lambda, N^{-1 / \alpha} \rho^{(N)}(\lambda)\right) \mathbf{1}_{\{0>\lambda>V(x)\}} \pi(\mathrm{d} x) \mathrm{d} \lambda .
\end{aligned}
$$

Consider the first term on the right-hand side of the above expression. Integrating over $x$, it equals

$$
N \int_{0}^{+\infty} \frac{\mathrm{d}}{\mathrm{d} \lambda} g\left(N^{-1 / \beta} \lambda, N^{-1 / \alpha} \rho^{(N)}(\lambda)\right) \pi[\lambda<V] \mathrm{d} \lambda .
$$

Making the change of variable $\lambda^{\prime}:=\lambda N^{1 / \beta}$ and letting $N \rightarrow+\infty$, we find that the limit equals

$$
c_{\beta}^{+} \int_{0}^{+\infty} \frac{\mathrm{d}}{\mathrm{d} \lambda} g\left(\lambda, \mathbf{1}_{[0, M]}(\lambda) \rho(\lambda)\right) \lambda^{-\beta} \mathrm{d} \lambda=\int_{0}^{+\infty} g\left(\lambda, \mathbf{1}_{[0, M]}(\lambda) \rho(\lambda)\right) v_{\beta}(\mathrm{d} \lambda) .
$$

The limit for the second term is computed in the same way and we obtain (A.16).

\section{A.2. Proof of (A.2)}

Since $\left\{X_{n}, n \geq 0\right\}$ is stationary and Markovian, it suffices only to show that, for $g$ as in the statement of Theorem A.1, we have

$$
\lim _{N \rightarrow+\infty} N \mathrm{E}\left(\mathrm{E}\left[g\left(Z_{1, N}\right) \mid g_{0}\right]\right)^{2}=0 .
$$

Let $\delta:=1 / 2 \operatorname{dist}(0, \operatorname{supp} g)>0$. We can estimate the expression under the limit as

$$
\begin{aligned}
N\|g\|_{\infty} \mathrm{E}\left(\mathrm{P}\left[\left|Z_{1, N}\right| \geq \delta \mid g_{0}\right]\right)^{2} \leq 2\|g\|_{\infty} & \left\{N \mathrm{E}\left(\mathrm{P}\left[\left|R_{0}\left(X_{1}, X_{0}\right)\right| \geq \frac{\delta N^{1 / \beta}}{2} \mid g_{0}\right]\right)^{2}\right. \\
& \left.+N \mathrm{E}\left(\mathrm{P}\left[\left|\tau^{(N)}\left(X_{1}\right)\right| \geq \frac{\delta N^{1 / \alpha}}{2} \mid g_{0}\right]\right)^{2}\right\} .
\end{aligned}
$$

The first term on the right-hand side of (A.21) can be estimated by

$$
2\|g\|_{\infty} N^{1-2 / \beta} \mathrm{E}\left(\mathrm{E}\left[\left|R_{0}\left(X_{1}, X_{0}\right)\right| \mid g_{0}\right]\right)^{2} \leq C N^{1-2 / \beta}\|P|\chi|\|_{L^{2}(\pi)}^{2}
$$

for some constant $C>0$ independent of $N$. The expression on the right-hand side tends to 0 as $N \rightarrow+\infty$, thanks to (A.12) and $\beta \in(1,2)$. 
The second term on the right-hand side of (A.21) can be estimated, using (A.13), by

$$
\begin{aligned}
2\|g\|_{\infty} N^{1-2 / \alpha} \mathrm{E}\left(\mathrm{E}\left[\tau^{(N)}\left(X_{1}\right) \mid g_{0}\right]\right)^{2} & \leq 2\|g\|_{\infty} N^{1-2 / \alpha}\left\|P \tau^{(N)}\right\|_{L^{2}(\pi)}^{2} \\
& \leq C N^{1-2 / \alpha} N^{2(1 / \alpha-1)} \\
& =\frac{C}{N}
\end{aligned}
$$

for some constants $C>0$ independent of $N$.

\section{A.3. Proof of (A.3)}

Using again stationarity and Doob's inequality for the martingale $\left\{Z_{t}^{(\Delta, N)}, t \geq 0\right\}$, the expression under the limit in (A.3) can be estimated from above by

$$
C T N \sum_{j=1}^{2}\left\{\mathrm{E}\left(Z_{1, N}^{(j)} \mathbf{1}_{\left\{\left|Z_{1, N}^{(i)}\right| \leq \Delta_{i}, i=1,2\right\}}\right)^{2}-\mathrm{E}\left(\mathrm{E}\left[Z_{1, N}^{(j)} \mathbf{1}_{\left\{\left|Z_{1, N}^{(i)}\right| \leq \Delta_{i}, i=1,2\right\}} \mid g_{0, N}\right]\right)^{2}\right\} .
$$

The term corresponding to $j=1$ can be estimated by, for some constants $C_{1}, C_{2}>0$,

$$
\begin{aligned}
C_{1} T N^{1-2 / \beta} \int \chi^{2}(x) \mathbf{1}_{\left\{|\chi(x)| \leq \Delta_{1} N^{1 / \beta}\right\}} \pi(\mathrm{d} x) & \leq C_{2} T N^{1-2 / \beta} \int_{0}^{\Delta_{1} N^{1 / \beta}} \lambda \pi(|\chi(x)| \geq \lambda) \mathrm{d} \lambda \\
& =C_{2} T N \int_{0}^{\Delta_{1}} \lambda \pi\left(|\chi(x)| \geq N^{1 / \beta} \lambda\right) \mathrm{d} \lambda .
\end{aligned}
$$

Using the fact that $\pi\left(|\chi(x)| \geq N^{1 / \beta} \lambda\right) \leq C_{3} N^{-1} \lambda^{-\beta}$ for some constant $C_{3}>0$, we conclude that the right-hand side of (A.22) can be estimated by

$$
C T \int_{0}^{\Delta_{1}} \lambda^{1-\beta} \mathrm{d} \lambda=C^{\prime} T \Delta_{1}^{2-\beta}
$$

for some constants $C, C^{\prime}>0$. A similar estimate can also be obtained for $j=2$. Estimate (A.3) then follows.

\section{Appendix B. The proof of Theorem 2.3}

In this section we retain the notation from the previous section. Assume furthermore that $\beta=2$. It is well known that, under the hypotheses made in Section 2.1, the components of the processes $\left\{X_{t}^{(N)}:=\left(S_{t}^{(N)}, T_{t}^{(N)}\right), t \geq 0\right\}$ converge in $\mathscr{D}$ (see, e.g. [18] and [24, Theorem 2, Chapter VII]) to a Brownian motion and an $\alpha$-stable subordinator process. This in turn implies tightness of the laws of $\left\{X_{t}^{(N)}, t \geq 0\right\}$ over $\mathscr{D} \times \mathscr{D}$ equipped with the uniform and $J_{1}$ topologies, respectively. To complete the proof of Theorem 2.3 we only need to show the weak convergence of finite-dimensional distributions of $\left\{X_{t}^{(N)}, t \geq 0\right\}$ to the respective finite-dimensional distribution of a Lévy process $\left\{\left(S_{t}, T_{t}\right), t \geq 0\right\}$ with exponent

$$
\psi\left(\xi_{1}, \xi_{2}\right):=\sigma^{2} \xi_{1}^{2} / 2+\int_{0}^{+\infty}\left(\mathrm{e}^{i \xi_{2} \lambda}-1\right) v_{\alpha}(\mathrm{d} \lambda)
$$

for some $\sigma \geq 0$. By a well-known Cramér-Wold device (see Theorem 9.5 of [11, p. 147]), it suffices to consider only one-dimensional distributions, i.e. to prove that, for $\left(\xi_{1}, \xi_{2}\right) \in \mathbb{R}^{2}$ and $t \geq 0$,

$$
\xi_{1} S_{t}^{(N)}+\xi_{2} T_{t}^{(N)} \Rightarrow \xi_{1} S_{t}+\xi_{2} T_{t} \quad \text { as } N \rightarrow+\infty
$$


For a fixed $M>0$, define $\bar{Z}_{N, t}^{(2)}(M):=\sum_{n=0}^{[N t]-1} \mathrm{E}\left[\tau^{(N)}\left(X_{n}\right) \mid g_{n-1, N}\right]$ with $\tau^{(N)}(x)$ given by (A.6). Using (A.2), we can argue, as in the proof of Equation (4.3) of [11], that

$$
\lim _{N \rightarrow+\infty} \bar{Z}_{N, t}^{(2)}(M)=t \int_{0}^{M} \lambda v_{\alpha}(\mathrm{d} \lambda)
$$

in probability. Since (A.8) is also in force for $\beta=2$, thanks to (B.1), it suffices only to show that, for a fixed $M>0$,

$$
\xi_{1} Z_{N, t}^{(1)}+\xi_{2} \tilde{Z}_{N, t}^{(2)}(M) \Rightarrow \xi_{1} S_{t}+\xi_{2} \tilde{T}_{t}(M) \text { as } N \rightarrow+\infty,
$$

where

$$
\tilde{Z}_{N, t}^{(2)}(M)=Z_{N, t}^{(2)}(M)-\bar{Z}_{N, t}^{(2)}(M),
$$

$Z_{N, t}^{(1)}$ and $Z_{N, t}^{(2)}(M)$ are given by (A.7), and $\left\{\left(S_{t}, \tilde{T}_{t}(M)\right), t \geq 0\right\}$ is a Lévy process with exponent

$$
\psi\left(\xi_{1}, \xi_{2}\right):=\frac{\sigma^{2} \xi_{1}^{2}}{2}+\int_{0}^{M}\left(\mathrm{e}^{i \xi_{2} \lambda}-1-i \xi_{2} \lambda\right) v_{\alpha}(\mathrm{d} \lambda)
$$

for some $\sigma>0$. In what follows we omit writing $M$ in the notation of the processes. Note that $\left\{\left(Z_{N, t}^{(1)}, Z_{N, t}^{(2)}\right), t \geq 0\right\}$ is a martingale with the increments given by

$$
\begin{aligned}
\tilde{Z}_{n, N} & =\left(\tilde{Z}_{n, N}^{(1)}, \tilde{Z}_{n, N}^{(2)}\right) \\
& :=\left(\frac{1}{N^{1 / 2}} R_{0}\left(X_{n}, X_{n-1}\right), \frac{1}{N^{1 / \alpha}}\left\{\tau^{(N)}\left(X_{n}\right)-\mathrm{E}\left[\tau^{(N)}\left(X_{n}\right) \mid g_{n-1, N}\right]\right\}\right) .
\end{aligned}
$$

We can therefore use the results of [8]. According to Theorem 1 of [8], in order to show (B.2), it suffices to prove the following result.

Proposition B.1. Suppose that $M_{-1, N}:=0$ and

$$
M_{n, N}:=\frac{\xi_{1}}{N^{1 / 2}} R_{0}\left(X_{n}, X_{n-1}\right)+\frac{\xi_{2}}{N^{1 / \alpha}}\left\{\tau^{(N)}\left(X_{n}\right)-\mathrm{E}\left[\tau^{(N)}\left(X_{n}\right) \mid g_{n-1, N}\right]\right\} \text { for } n \geq 0 .
$$

Then, for any $a<b$, we have

$$
\lim _{N \rightarrow+\infty} \sum_{n=1}^{[N t]-1} \mathrm{E}\left[M_{n, N}^{2} \mathbf{1}_{\left\{a<M_{n, N}<b\right\}} \mid g_{n-1, N}\right]=t\left[G_{\xi_{1}, \xi_{2}}(b)-G_{\xi_{1}, \xi_{2}}(a)\right]
$$

in probability, where the function $G_{\theta_{1}, \theta_{2}}(\cdot)$ is given by $G_{\xi_{1}, \xi_{2}}(\lambda)=0, \lambda<0$, and

$$
G_{\xi_{1}, \xi_{2}}(\lambda)=\sigma^{2} \xi_{1}^{2}+c_{\alpha}\left|\xi_{2}\right|^{\alpha} \lambda^{1-\alpha}
$$

for $\lambda>0$ and some $\sigma>0$.

Proof. The proof is carried out in two steps. First we prove that, for any interval $(a, b)$ that does not contain 0 and any $C^{\infty}$ function $g$ supported in that interval, we have

$$
\lim _{N \rightarrow+\infty} \sum_{n=0}^{[N t]-1} \mathrm{E}\left[g\left(M_{n, N}\right) \mid g_{n-1, N}\right]=t\left|\xi_{2}\right|^{\alpha} \int_{0}^{M} g(\lambda) v_{\alpha}(\mathrm{d} \lambda)
$$

in probability. The argument is essentially a repetition of the proof of (A.2) in the previous 
section so we omit it. Next, we show that, for any $c>0$,

$$
\limsup _{N \rightarrow+\infty} \mathrm{E}\left|\sum_{n=0}^{[N t]-1} \mathrm{E}\left[M_{n, N}^{2} \mathbf{1}_{\left\{\left|M_{n, N}\right|<c\right\}} \mid g_{n-1}\right]-\frac{1}{2} \sigma^{2} t \xi_{1}^{2}\right|=h(c),
$$

where $\lim _{c \rightarrow 0+} h(c)=0$. For an arbitrary interval $(a, b)$, where $a<0<b$, we divide it into a sum of three disjoint intervals, $(a,-c),(-c, c)$, and $(c, b)$, where $0<c<\min [-a, b]$, and conclude using the above results and a standard approximation argument that

$$
\limsup _{N \rightarrow+\infty} \mathrm{E}\left|\sum_{n=0}^{[N t]-1} \mathrm{E}\left[M_{n, N}^{2} \mathbf{1}_{\left\{a<M_{n, N}<b\right\}} \mid g_{n-1, N}\right]-t\left[G_{\xi_{1}, \xi_{2}}(b)-G_{\xi_{1}, \xi_{2}}(a)\right]\right|=0 .
$$

Proof of (B.3). Suppose that $c>0$ is arbitrary. We consider only the case when both $\xi_{1}, \xi_{2} \neq 0$. The other cases can be proved by adjusting (and simplifying) the argument. Note that

$$
\begin{aligned}
\sum_{n=0}^{[N t]-1} \mathrm{E}\left[\left(M_{n, N}\right)^{2} \mathbf{1}_{\left\{\left|M_{n, N}\right|<c\right\}} \mid g_{n-1, N}\right]= & \xi_{1}^{2} \sum_{n=0}^{[N t]-1} \mathrm{E}\left[\left(\tilde{Z}_{n, N}^{(1)}\right)^{2} \mathbf{1}_{\left\{\left|M_{n, N}\right|<c\right\}} \mid g_{n-1, N}\right] \\
& +\xi_{2}^{2} \sum_{n=0}^{[N t]-1} \mathrm{E}\left[\left(\tilde{Z}_{n, N}^{(2)}\right)^{2} \mathbf{1}_{\left\{\left|M_{n, N}\right|<c\right\}} \mid g_{n-1, N}\right] \\
& +2 \xi_{1} \xi_{2} \sum_{n=0}^{[N t]-1} \mathrm{E}\left[\tilde{Z}_{n, N}^{(1)} \tilde{Z}_{n, N}^{(2)} \mathbf{1}_{\left\{\left|M_{n, N}\right|<c\right\}} \mid g_{n-1, N}\right]
\end{aligned}
$$

Denote the terms appearing on the right-hand side by $U_{N}, V_{N}$, and $W_{N}$, respectively. For an appropriate constant $C>0$, we have

$$
\begin{aligned}
& \mathrm{E}\left|W_{N}\right| \leq C\left\{N\left(\mathrm{E}\left[\left(\xi_{1} \tilde{Z}_{1, N}^{(1)}\right)^{2}\right]\right)^{1 / 2}\left\{\mathrm{E}\left[\left(\xi_{2} \tilde{Z}_{1, N}^{(2)}\right)^{2}\right],\left|\xi_{2} \tilde{Z}_{1, N}^{(2)}\right|<10 c\right\}^{1 / 2}\right. \\
&\left.+N\left|\xi_{1} \xi_{2}\right| \mathrm{E}\left[\left|\tilde{Z}_{1, N}^{(1)} \tilde{Z}_{1, N}^{(2)}\right|,\left|\xi_{1} \tilde{Z}_{1, N}^{(1)}\right|>9 c,\left|\xi_{2} \tilde{Z}_{1, N}^{(2)}\right| \geq 10 c\right]\right\} .
\end{aligned}
$$

Denote the first and second terms appearing in the braces on the right-hand side by $W_{N}^{(1)}$ and $W_{N}^{(2)}$, respectively. We have

$$
\mathrm{P}\left[\left|\xi_{2} \tilde{Z}_{n, N}^{(2)}\right|>\lambda\right] \leq \pi\left[\tau>\frac{N^{1 / \alpha} \lambda}{2\left|\xi_{2}\right|}\right]+\pi\left[P \tau^{(N)}>\frac{N^{1 / \alpha} \lambda}{2\left|\xi_{2}\right|}\right] .
$$

The first term on the right-hand side is clearly less than or equal to $C N^{-1} \lambda^{-\alpha}$ for all $\lambda>0$, $N \geq 1$, and a certain constant $C>0$, independent of $n \geq 0$. By the Markov inequality and (A.13), the second term can be estimated by $\lambda^{-1} N^{-1 / \alpha}\|P \tau\|_{L^{2}(\pi)} \leq C(\lambda N)^{-1}$. We can deduce that

$$
\mathrm{P}\left[\left|\xi_{2} \tilde{Z}_{n, N}^{(2)}\right|>\lambda\right] \leq C(N \lambda)^{-1}\left(1+\lambda^{1-\alpha}\right)
$$

for all $\lambda>0, N \geq 1$, and a certain constant $C>0$, independent of $n, N \geq 0$. Using (B.4) and the elementary estimate $\left\{\mathrm{E}\left[\left[\xi_{1} \tilde{Z}_{1, N}^{(1)}\right]^{2}\right]\right\}^{1 / 2} \leq C N^{-1 / 2}\|\chi\|_{L^{2}(\pi)}$, we obtain

$$
W_{N}^{(1)} \leq C\|\chi\|_{L^{2}(\pi)}\left[\int_{0}^{10 c}\left(1+\lambda^{1-\alpha}\right) \mathrm{d} \lambda\right]^{1 / 2} \leq C^{\prime}\|\chi\|_{L^{2}(\pi)}\left[c\left(1+c^{1-\alpha}\right)\right]^{1 / 2}
$$

for some constants $C, C^{\prime}>0$. 
On the other hand, using Chebyshev's inequality, we obtain

$$
\mathrm{P}\left[\left|\xi_{1} \tilde{Z}_{n, N}^{(1)}\right|>\lambda\right] \leq \frac{C\|\chi\|_{L^{2}(\pi)}^{2}}{N \lambda^{2}}
$$

for all $\lambda>0$. The constant $C>0$ appearing here does not depend on $N, n$, and $\lambda$. Thus, for some constants $C, C^{\prime}>0$, we have

$$
\begin{aligned}
W_{N}^{(2)} & \leq C N M \mathrm{E}\left[\left|\xi_{1} \tilde{Z}_{1, N}^{(1)}\right|,\left|\xi_{1} \tilde{Z}_{1, N}^{(1)}\right|>9 c\right] \\
& \leq C N M\left\{\mathrm{E}\left[\left(\xi_{1} \tilde{Z}_{1, N}^{(1)}\right)^{2},\left|\xi_{1} \tilde{Z}_{1, N}^{(1)}\right|>9 c\right]\right\}^{1 / 2} \mathrm{P}^{1 / 2}\left[\left|\xi_{1} \tilde{Z}_{1, N}^{(1)}\right|>9 c\right] \\
& \stackrel{(\text { B.5) }}{\leq} C^{\prime}\left\{\mathrm{E}\left[R_{0}^{2}\left(X_{1}, X_{0}\right),\left|\xi_{1} \tilde{Z}_{1, N}^{(1)}\right|>9 c\right]\right\}^{1 / 2} \\
& \rightarrow 0
\end{aligned}
$$

as $N \rightarrow+\infty$. We have proved therefore that $\lim _{\sup } \operatorname{sut}_{N \rightarrow \infty} \mathrm{E}\left|W_{N}\right| \leq C\left[c\left(1+c^{1-\alpha}\right)\right]^{1 / 2}$, where $c>0$ can be chosen arbitrarily. Thus, $\lim _{N \rightarrow+\infty} \mathrm{E}\left|W_{N}\right|=0$.

Note that

$$
\begin{aligned}
\mathrm{E}\left|V_{N}\right| \leq & C N \mathrm{E}\left[\left(\xi_{2} \tilde{Z}_{n, N}^{(2)}\right)^{2},\left|\xi_{2} \tilde{Z}_{n, N}^{(2)}\right|<10 c\right] \\
& +C N^{1-2 / \alpha} \mathrm{E}\left[\left(\tau^{(N)}\left(X_{0}\right)\right)^{2},\left|\xi_{1} \tilde{Z}_{n, N}^{(1)}\right|>9 c,\left|\xi_{2} \tilde{Z}_{n, N}^{(2)}\right| \geq 10 c\right]
\end{aligned}
$$

for some constant $C>0$. Denote the first and second terms on the right-hand side of (B.6) by $V_{N}^{(1)}$ and $V_{N}^{(2)}$, respectively. Estimating in the same way as in (B.4), we deduce that $V_{N}^{(1)} \leq C\left[c\left(1+c^{1-\alpha}\right)\right]^{1 / 2}$ for some constant $C>0$. This term can be made arbitrarily small by choosing a sufficiently small $c>0$. On the other hand, from Chebyshev's inequality for some constants $C, C^{\prime}>0$, we have

$$
\begin{aligned}
V_{N}^{(2)} & \leq C N M^{2} \mathrm{P}\left[\left|\xi_{1} R_{0}\left(X_{1}, X_{0}\right)\right| \geq 9 c N^{1 / 2}\right] \\
& \leq C^{\prime} M^{2} \mathrm{E}\left[\left(\xi_{1} R_{0}\left(X_{1}, X_{0}\right)\right)^{2},\left|\xi_{1} R_{0}\left(X_{1}, X_{0}\right)\right| \geq 9 c N^{1 / 2}\right] \\
& \rightarrow 0
\end{aligned}
$$

both a.s. and in the $L^{1}$ sense, as $N \rightarrow+\infty$. Finally, we can write that $U_{N}=\hat{U}_{N}-\bar{U}_{N}$, where

$$
\hat{U}_{N}:=\xi_{1}^{2} \sum_{n=0}^{[N t]-1} \mathrm{E}\left[\left(\tilde{Z}_{n, N}^{(1)}\right)^{2} \mid g_{n-1, N}\right]
$$

and

$$
U_{N}:=\xi_{1}^{2} \sum_{n=1}^{[N t]} \mathrm{E}\left[\left(\tilde{Z}_{n, N}^{(1)}\right)^{2} \mathbf{1}_{\left\{\left|\tilde{Z}_{n, N}\right|>c\right\}} \mid g_{n-1, N}\right] .
$$

By the ergodic theorem,

$$
\hat{U}_{N}=\frac{\xi_{1}^{2}}{N} \sum_{n=0}^{[N t]-1}\left[P V^{2}\left(X_{n-1}\right)+P \chi^{2}\left(X_{n-1}\right)-(P \chi)^{2}\left(X_{n-1}\right)\right] \rightarrow \frac{1}{2} \sigma^{2} \xi_{1}^{2} t \quad \text { as } N \rightarrow+\infty
$$

both a.s. and in the $L^{1}$ sense. Here

$$
\sigma^{2}:=2\left(\|V\|_{L^{2}(\pi)}^{2}+\|\chi\|_{L^{2}(\pi)}^{2}-\|P \chi\|_{L^{2}(\pi)}^{2}\right) .
$$


Using stationarity, we deduce that, for a certain constant $C>0$,

$$
\mathrm{E}\left|\bar{U}_{N}\right| \leq C \xi_{1}^{2} \mathrm{E}\left[R_{0}^{2}\left(X_{1}, X_{0}\right),\left|\xi_{1} R_{0}\left(X_{1}, X_{0}\right)\right|>\frac{c N^{1 / 2}}{2} \text { or }\left|\xi_{2} \tilde{Z}_{1, N}^{(2)}\right|>\frac{c}{2}\right] \rightarrow 0
$$

as $N \rightarrow+\infty$. The convergence follows from the $L^{2}$-integrability of $R_{0}\left(X_{1}, X_{0}\right)$ and (B.4).

\section{Acknowledgements}

The authors would like to express their thanks to an anonymous referee for pointing out that in an earlier version of this paper the result concerning the case when the limiting process admits common jumps was erroneously formulated. The work of T. K. was partially supported by the Polish MNiSW grant NN 201419139 and the EC FP6 Marie Curie ToK programme SPADE2, under grants MTKD-CT-2004-014508 and Polish MNiSW SPB-M. The work of M. J. was partially supported by Agence Nationale de la Recherche, under grant ANR-07BLAN-2-184264 (LHMSHE).

\section{References}

[1] Athreya, K. B. (1986). Darling and Kac Revisited. Sankhyā A 48, 255-266.

[2] Becker-Kern, P., Meerschaert, M. M. and Scheffler, H.-P. (2004). Limit theorems for coupled continuous time random walks. Ann. Prob. 32, 730-756,

[3] Becker-Kern, P., Meerschaert, M. M. and Scheffler, H.-P. (2011). Correction to 'Limit theorem for coupled continuous time random walks'. To appear in Ann. Prob. Available at http://www.imstat.org/aop/ future_papers.htm.

[4] Benson, D., Wheatcraft, S. and Meerschaert, M. (2000). Application of a fractional advection-dispersion equation. Water Resources Res. 36, 1403-1412.

[5] Benson, D., Wheatcraft, S. And Meerschaert, M. (2000). The fractional-order governing equation of Lévy motion. Water Resources Res. 36, 1413-1424.

[6] Billingsley, P. (1999). Convergence of Probability Measures, 2nd edn. John Wiley, New York.

[7] Breiman, L. (1968). Probability. Addison-Wesley, Reading, MA.

[8] Brown, B. M. and Eagleson, G. K. (1971). Martingale convergence to infinite divisible laws with finite variances. Trans. Amer. Math. Soc. 162, 449-453.

[9] Clark, J., De Roeck, W. And Maes, C. (2009). Diffusive behavior from a quantum master equation. Preprint.

[10] Darling, D. A. And Kac, M. (1957). On occupation times for Markoff processes. Trans. Amer. Math. Soc. 84, 444-458.

[11] Durrett, R. and Resnick, S. I. (1978). Functional limit theorems for dependent variables. Ann. Prob. 6, 829-846.

[12] Foguel, S. R. (1969). The Ergodic Theory of Markov Processes. Van Nostrand Reinhold, New York.

[13] Gorenflo, R. ANd Mainardi, F. (2003). Fractional diffusion processes: probability distributions and continuous time random walk. In Processes with Long Range Correlations (Lecture Notes Phys. 621), Springer, Berlin, pp. 148-166.

[14] Gorenflo, R., Mainardi, F., Scalas, E. and Raberto, M. (2001). Fractional calculus and continuous-time finance. III. The diffusion limit. In Mathematical Finance (Konstanz, 2000), Birkhäuser, Basel, pp. 171-180.

[15] Harris, T. E. (1956). The existence of stationary measures for certain Markov processes. In Proc. 3rd Berkeley Symp. Mathematical Statist. Prob., Vol. II, University of California Press, Berkeley, pp. 113-124.

[16] Höpfner, R. AND Löcherbach, E. (2003). Limit theorems for null recurrent Markov processes. Mem. Amer. Math. Soc. 161, 92pp.

[17] JAMison, B. AND OREY, S. (1967). Markov chains recurrent in the sense of Harris. Z. Wahrscheinlichkeitsth. 8, 41-48.

[18] Jara, M., Komorowski, T. And Olla, S. (2009). Limit theorems for additive functionals of a Markov chain. Ann. Appl. Prob. 19, 2270-2300.

[19] Jurlewicz, A., Kern, P., Meerschaert, M. M. And Scheffler, H.-P. (2010). Oracle continuous time random walks. Preprint. Available at http://www.uni-siegen.de/fb6/src/scheffler/research/octrw2.pdf.

[20] Kolokol'tsov, V. N. (2009). Generalized continuous-time random walks, subordination by hitting times, and fractional dynamics. Theory Prob. Appl. 53, 594-609. 
[21] Mainardi, F., Raberto, M., Gorenflo, R. and Scalas, E. (2000). Fractional calculus and continuous-time finance II: the waiting-time distribution. Physica A 287, 468-481.

[22] Meerschaert, M. M. And Scheffler, H.-P. (2008). Triangular array limits for continuous time random walks. Stoch. Process. Appl. 118, 1606-1633.

[23] Montroll, E. W. And Weiss, G. H. (1965). Random walks on lattices. II. J. Math. Phys. 6, 167-181.

[24] Rosenblatt, M. (1971). Markov Processes. Structure and Asymptotic Behavior. Springer, New York.

[25] Sato, K. I. (1999). Lévy Processes and Infinitely Divisible Distributions. Cambridge University Press.

[26] Shlesinger, M., Klafter, J. And Wong, Y. M. (1982). Random walks with infinite spatial and temporal moments. J. Statist. Phys. 27, 499-512.

[27] Shlesinger, M. F., Zaslavsky, G. M. and Klafter, J. (1993). Strange kinetics. Nature 363, $31-37$.

[28] Straka, P. AND Henry, B. I. (2011). Lagging and leading coupled continuous time random walks, renewal times and their joint limits. Stoch. Process. Appl. 121, 324-336.

[29] Whitt, W. (2002). Stochastic-Process Limits. Springer, New York.

[30] Zaslavsky, G. M. (2002). Chaos, fractional kinetics, and anomalous transport. Phys. Rep. 371, 461-580. 